\title{
ABUNDANCE ANOMALIES IN CP CRUCIS (NOVA CRUX 1996)
}

\author{
James E. Lyke, ${ }^{1}$ X. P. Koenig,,${ }^{2,3}$ M. J. Barlow, ${ }^{2}$ R. D. Gehrz,${ }^{1}$ Charles E. Woodward, ${ }^{1}$ \\ Sumner Starrfield ${ }^{4}$ D. Péquignot, ${ }^{5}$ A. Evans, ${ }^{6}$ A. Salama,${ }^{7}$ R. González-Riestra, ${ }^{8}$ \\ Matthew A. Greenhouse, ${ }^{9}$ R. M. Huellming, ${ }^{10}$ Terry J. Jones, ${ }^{1}$ Johchim Krautter,${ }^{11}$ \\ H. B. Ögelman, ${ }^{12}$ R. Mark Wagner, ${ }^{13}$ S. L. Lumsden, ${ }^{14}$ and R. E. Williams ${ }^{15}$ \\ Received 2003 February 7; accepted 2003 April 22
}

\begin{abstract}
We present spectroscopic observations of the classical nova CP Crucis (Nova Crux 1996) obtained with the Infrared Space Observatory Short Wavelength Spectrometer (ISO SWS) and the Anglo-Australian Telescope using both the Infrared Imaging Spectrograph and the Royal Greenwich Observatory Spectrograph. From the expansion parallax, we find that $\mathrm{CP}$ Crucis lies at a distance of $2.6 \pm 0.5 \mathrm{kpc}$ and reached a maximum $M_{V}$ of -8.7 at 0.96 days after discovery. We find abundance enhancements versus solar by mass of 75, 17, and 27 for $\mathrm{N}, \mathrm{O}$, and $\mathrm{Ne}$, respectively. Additionally, we constrain the $\mathrm{Mg}$ abundance in the ejecta to be approximately solar. Abundance analysis suggests CP Crucis is an old Population I binary system. Combining the strong $\mathrm{N}$ and $\mathrm{Ne}$ abundances with the relatively low $\mathrm{Mg}$ abundance and a $\mathrm{Ne} / \mathrm{O}$ ratio of 0.5 , we propose that $\mathrm{CP}$ Crucis is an example of the "missing link" between $\mathrm{CO}$ and $\mathrm{ONeMg}$ novae.

Key words: circumstellar matter — novae, cataclysmic variables stars: individual (Nova Crux 1996, CP Crucis)
\end{abstract}

\section{INTRODUCTION}

CP Crucis (Nova Crux 1996; CP Cru) was discovered on 1996 August 26.04 UT (JD 2,450,321.54) by W. Liller (Liller, Overbeek, \& Mattei 1996). As the last prediscovery image of the region was taken about 2 weeks before the discovery, we define day 0 as the discovery date. Recently, Woudt \& Warner (2003) have shown CP Cru to be an eclipsing binary with $P_{\mathrm{orb}}=22.7 \mathrm{hr}$. Figure 1 shows a composite visual light curve compiled from IAU Circulars and the American Association of Variable Star Observers (AAVSO; Liller et al. 1996; Monard, Nelson, \& Farrell 1996; Garrad 1996; J. A. Mattei 2002, private communication). ${ }^{16} \mathrm{We}$

\footnotetext{
${ }^{1}$ Department of Astronomy, University of Minnesota, 116 Church Street, SE, Minneapolis, MN 55455.

${ }^{2}$ Department of Physics and Astronomy, University College London, Gower Street, London WC1E 6BT, UK.

${ }^{3}$ Current address: Harvard-Smithsonian Center for Astrophysics, 60 Garden Street, Cambridge, MA 02138.

${ }^{4}$ Department of Physics and Astronomy, Arizona State University, Tempe, AZ 85287.

${ }^{5}$ Observatoire de Paris-Meudon, Place Jules Janssen 5, F-92195 Meudon Cedex, France.

${ }^{6}$ Department of Physics, Keele University, Keele, Staffordshire, ST5 $5 \mathrm{BG}, \mathrm{UK}$

${ }^{7}$ ISO Science Operations Centre, Astrophysics Division of ESA, Apdo. 50727, E-28080 Madrid, Spain.

${ }^{8}$ ESA Villafranca del Castillo Satellite Tracking Station, Apdo. 50727, E-28080 Madrid, Spain.

${ }^{9}$ NASA Goddard Space Flight Center, Code 685, Greenbelt, MD 20771 .

${ }^{10}$ Deceased 2000 July.

${ }^{11}$ Landessternwarte, Königstuhl, D-69117 Heidelberg, Germany.

12 Department of Astronomy, University of Wisconsin-Madison, 475 North Charter Street, Madison, WI 53706.

${ }^{13}$ Large Binocular Telescope Observatory, University of Arizona, 933 North Cherry Avenue, Tucson, AZ 85721.

${ }^{14}$ Department of Physics and Astronomy, University of Leeds, Leeds LS2 9JT, UK.

${ }^{15}$ Space Telescope Science Institute, 3700 San Martin Drive, Baltimore, MD 21218.

${ }^{16}$ Observations from the AAVSO International Database; see http://aavso.org/adata/database.stm.
}

determine that the time for the observed visual light curve to decline by 2 and 3 mag from maximum light, respectively, is $t_{2}=4.3 \pm 0.7$ days and $t_{3}=10 \pm 2$ days, classifying CP Cru as a very fast nova (Payne-Gaposchkin 1964). Spectra from 1997 January 10.3 UT reported by Della Valle \& Benetti (1997) found rapidly expanding ejecta with an expansion velocity, $v_{\text {exp }}$, provided by the FWHM of $\mathrm{H} \alpha$ and $\mathrm{H} \beta$ of $v_{\exp } \sim 2000 \mathrm{~km} \mathrm{~s}^{-1}$. These authors also suggest that, because of the strength of the He II $\lambda 4686$ line, CP Cru belongs to the $\mathrm{He} / \mathrm{N}$ class of novae (Williams 1992). Furthermore, given the Galactic plane position of $\mathrm{CP}$ Cru $\left(l=298^{\circ} .11, b=0.74\right)$, the $\mathrm{He} / \mathrm{N}$ nova classification also agrees with the assertion of Della Valle \& Livio (1998) that $\mathrm{He} / \mathrm{N}$ novae tend to be concentrated in the disk.

Spectroscopic observations of classical novae can be used to determine the chemical composition of their ejecta and reveal the chemical enrichment that occurred during the lifetime of the progenitor (Gehrz et al. 1998). Moreover, spectra of classical novae enable one to study the dynamical development of novae ejecta through line profile fitting. Infrared (IR) observations are critical when interstellar or circumstellar extinction is strong. Generally, 100-200 days after outburst novae develop strong IR forbidden line emission. However, as we shall see, CP Cru developed strong [Ne II] $12.81 \mu \mathrm{m}$ line emission within 24 days of outburst. Forbidden line transitions, such as [Ne II], provide a strong cooling source for the low-density astrophysical plasma ejected by the thermonuclear runaway event (Osterbrock 1989).

CP Cru represents the third in a series of classical novae we observed with both the Infrared Space Observatory Short Wavelength Spectrograph (ISO SWS) and complementary ground-based telescopes (V1425 Aql: Lyke et al. 2001, hereafter Paper I; V723 Cas: Evans et al. 2003). CP Cru has a southern declination and was relatively faint at maximum. Consequently, to date, little discussion of this object has appeared in the literature. Here we present the visual light curve, optical and IR spectroscopic measurements, and abundance estimates for the ejecta of CP Cru. 


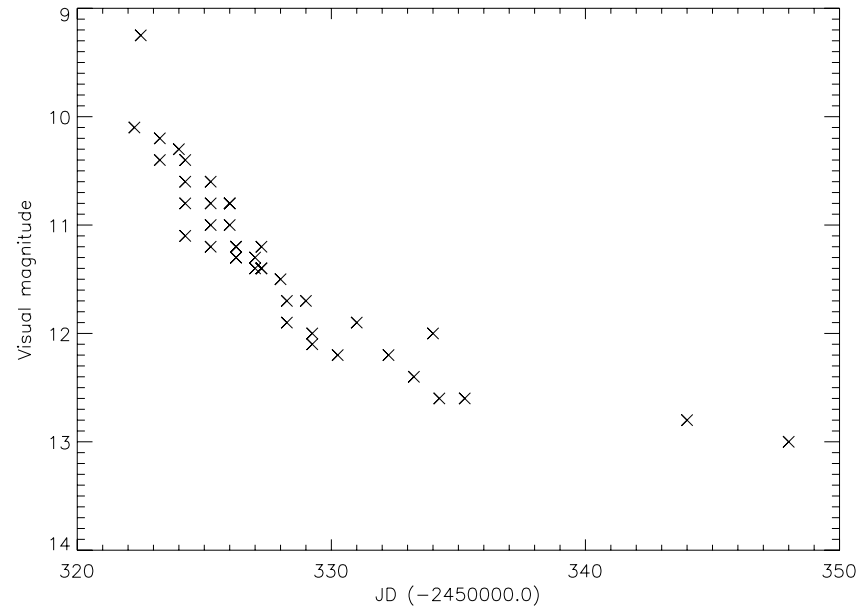

FIG. 1.-Visual light curve from observations reported in IAU Circulars and to the AAVSO. From these data, we determine $t_{2}=4.3 \pm 0.7$ days and $t_{3}=10 \pm 2$ days.

\section{OBSERVATIONS AND DATA REDUCTION}

We adopted as our pointing coordinates for CP Cru the astrometric position determined by Garradd (1996) of

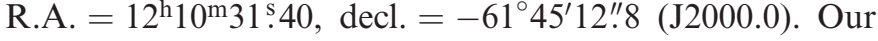
spectroscopic observations of CP Cru covered days 24-334 after the outburst in optical and IR wavelengths (see Table 1).

\subsection{ISO SWS}

CP Cru was observed with ISO SWS using the Astronomical Observing Template (AOT) SWS-01. This AOT yielded fast, full-range scans at a resolution of $\lambda / \Delta \lambda \simeq 2000$, corresponding to a velocity resolution of $150 \mathrm{~km} \mathrm{~s}^{-1}$ (Valentijn et al. 1996). The ISO SWS instrument is described in detail in de Graauw et al. (1996). The ISO SWS data were reduced using version 10.1 of the ISO SWS pipeline and analyzed from the Auto Analysis Result (AAR) level in the Observer's SWS Interactive Analysis (OSIA) and the ISO Spectral Analysis Package (ISAP), version 2.1 (Sturm 1997). The full range of each spectrum is $2.38-45.2 \mu \mathrm{m}$ and is divided into four bands of 12 detectors each. Data flagged as "Bad " by the ISO SWS pipeline were removed by an ISAP task. AOT SWS-01 scanned the grating twice (up/down scans) to produce two data sets for each of the 48 detectors. This allowed us to distinguish real features (that appeared in both the up and down scans) from transient noise events such as cosmic rays. The data were smoothed to approximately $2-5$ times the resolution element since SWS spectra are oversampled. Single component Gaussians were fitted to the averaged lines from which fluxes, line centers, and FWHM velocities were obtained and are reported in Table 2.

\subsection{IRIS}

Infrared Imaging Spectrograph (IRIS) observations on the $3.9 \mathrm{~m}$ Anglo-Australian Telescope (AAT) were obtained with the cross-dispersed $I J$ and $H K$ echelles at the f/36 focus, yielding a resolution of $\lambda / \Delta \lambda \simeq 400$ with the narrow slit. Both narrow- and wide-slit spectra were taken on days 91.7 and 179.6 to give both higher signal-to-noise ratio $(\mathrm{S} / \mathrm{N})$ and more robust flux calibration, respectively. Only narrow-slit spectra were obtained on day 266.4. The narrow slit is $1 . " 4$ wide, $13^{\prime \prime}$ long; the wide slit is 5 ".8 wide, $13^{\prime \prime}$ long. The IRIS data were extracted and flux calibrated using standard methods and the FIGARO data reduction system

TABLE 1

ObServational Details

\begin{tabular}{|c|c|c|c|c|c|}
\hline Description & $\begin{array}{l}\text { Wavelength } \\
\text { Range }^{\mathrm{a}}\end{array}$ & Date & $\begin{array}{l}\text { Time } \\
\text { (UT) }\end{array}$ & $\begin{array}{l}\text { Exposure } \\
\text { (s) }\end{array}$ & $\begin{array}{c}\text { Days since } \\
\text { Outburst }^{\mathrm{b}}( \pm 4)\end{array}$ \\
\hline ISO SWS- $01^{\mathrm{c}} \ldots \ldots \ldots$ & $2.38-45.2$ & 1996 Sep 19 & $15: 58$ & 6538 & 24.7 \\
\hline AAT IRIS $H K \mathrm{~N}^{\mathrm{d}}$. & $1.4-2.5$ & 1996 Nov 25 & $17: 36$ & 960 & 91.7 \\
\hline AAT IRIS $H K \mathrm{~W}^{\mathrm{d}}$. & $1.4-2.5$ & 1996 Nov 25 & $18: 29$ & 60 & 91.7 \\
\hline AAT IRIS $I J \mathrm{~N} \ldots \ldots$. & $0.85-1.5$ & 1996 Nov 25 & 18:04 & 960 & 91.7 \\
\hline AAT IRIS $I J \mathrm{~W}$. & $0.85-1.5$ & 1996 Nov 25 & $18: 25$ & 60 & 91.7 \\
\hline AAT IRIS $H K \mathrm{~N} \ldots \ldots \ldots \ldots \ldots$ & $1.4-2.5$ & 1997 Feb 21 & $14: 24$ & 1200 & 179.6 \\
\hline AAT IRIS $H K \mathrm{~W} \ldots \ldots \ldots \ldots \ldots$ & $1.4-2.5$ & 1997 Feb 21 & $14: 48$ & 480 & 179.6 \\
\hline AAT IRIS $I J \mathrm{~N} \ldots \ldots \ldots \ldots \ldots$ & $0.85-1.5$ & 1997 Feb 21 & $15: 03$ & 1200 & 179.6 \\
\hline AAT IRIS $I J \mathrm{~W} \ldots \ldots \ldots \ldots \ldots . . . . .$. & $0.85-1.5$ & 1997 Feb 21 & $16: 24$ & 600 & 179.6 \\
\hline 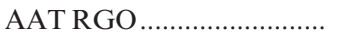 & $3357-9000$ & 1997 Mar 21 & $15: 23$ & 1100 & 207.6 \\
\hline 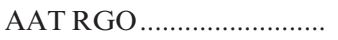 & $3357-9000$ & 1997 Mar 21 & $15: 46$ & 500 & 207.6 \\
\hline 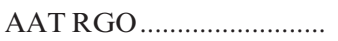 & $3357-9000$ & 1997 Mar 21 & $16: 19$ & 1000 & 207.6 \\
\hline 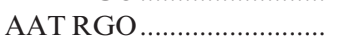 & $3357-9000$ & 1997 Mar 21 & $16: 38$ & 500 & 207.6 \\
\hline AAT IRIS $H K \mathrm{~N} \ldots \ldots \ldots \ldots \ldots$ & $1.4-2.5$ & 1997 May 19 & $08: 48$ & 2160 & 266.4 \\
\hline AAT IRIS $I J \mathrm{~N} \ldots \ldots \ldots \ldots \ldots \ldots$ & $0.85-1.5$ & 1997 May 19 & 09:54 & 1800 & 266.4 \\
\hline 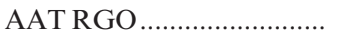 & $3233-9253$ & 1997 Jun 7 & 11:08 & 1560 & 285.5 \\
\hline 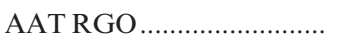 & $3233-9253$ & 1997 Jun 7 & $11: 50$ & 900 & 285.5 \\
\hline AAT RGO ............................ & $3233-9253$ & 1997 Jun 7 & $12: 55$ & 900 & 285.5 \\
\hline 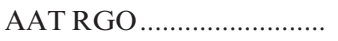 & $3233-9253$ & 1997 Jun 7 & $13: 12$ & 600 & 285.5 \\
\hline ISO SWS-01.......................... & $2.38-45.2$ & 1997 Jun 22 & 01:00 & 6537 & 300.0 \\
\hline ISO SWS-01 ......................... & $2.38-45.2$ & 1997 Jul 26 & $06: 38$ & 6538 & 334.2 \\
\hline
\end{tabular}

a Wavelength units are microns for ISO SWS and AAT IRIS and angstroms for AAT RGO

${ }^{\mathrm{b}}$ Day 0 is determined by Liller et al. 1996.

c SWS AOTs are described in de Graauw et al. 1996.

${ }^{\mathrm{d}} \mathrm{N}=$ narrow slit, $\mathrm{W}=$ wide slit. 
TABLE 2

ISO SWS EMISSION Line Data on Days 24.7, 300, AND 334

\begin{tabular}{|c|c|c|c|c|}
\hline $\begin{array}{c}\lambda \\
(\mu \mathrm{m})\end{array}$ & Identity & Observed $F_{\lambda} / F_{\mathrm{Br} \alpha}{ }^{\mathrm{a}}$ & $\begin{array}{l}\text { FWHM } \\
\left(\mathrm{km} \mathrm{s}^{-1}\right)\end{array}$ & Dereddened $F_{\lambda} / F_{\mathrm{Br} \alpha}{ }^{\mathrm{b}}$ \\
\hline \multicolumn{5}{|c|}{ Day 24.7} \\
\hline 2.6252 . & $\mathrm{Br} \beta$ & 0.671 & 3140 & 0.833 \\
\hline $2.6744 \ldots \ldots \ldots \ldots \ldots$ & Pf $13-5$ & 0.100 & 3260 & 0.122 \\
\hline $2.7575 \ldots \ldots \ldots \ldots$ & Pf $12-5$ & 0.090 & 3425 & 0.108 \\
\hline $2.8722 \ldots \ldots \ldots \ldots \ldots$ & Pf $11-5$ & 0.093 & 3236 & 0.109 \\
\hline $2.909 \ldots \ldots \ldots \ldots \ldots$ & $?$ & 0.041 & 2886 & 0.048 \\
\hline 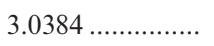 & Pf $10-5$ & 0.124 & $\ldots$ & 0.140 \\
\hline $3.0909 \ldots \ldots \ldots \ldots \ldots$ & He II 7-6? & 0.059 & $\ldots$ & 0.066 \\
\hline $3.2961 \ldots \ldots \ldots \ldots . .$. & Pf $9-5$ & 0.146 & 2956 & 0.159 \\
\hline $3.7395 \ldots \ldots \ldots \ldots \ldots$ & Pf $8-5$ & 0.162 & 3167 & 0.167 \\
\hline $4.0512 \ldots \ldots \ldots \ldots \ldots$ & $\operatorname{Br} \alpha$ & 1.000 & 3330 & 1.000 \\
\hline $4.2945 \ldots \ldots \ldots \ldots \ldots$ & He I & 0.072 & $\ldots$ & 0.071 \\
\hline $4.6525 \ldots \ldots \ldots \ldots \ldots$ & $\operatorname{Pf} \beta$ & 0.263 & 3351 & 0.253 \\
\hline $5.1273 \ldots \ldots \ldots \ldots \ldots$ & $\mathrm{Hu} 10-6$ & 0.106 & $\ldots$ & 0.100 \\
\hline $5.9066 \ldots \ldots \ldots \ldots \ldots$ & Hu 9-6 & 0.117 & 3578 & 0.108 \\
\hline $7.4578 \ldots \ldots \ldots \ldots \ldots$ & $\operatorname{Pf} \alpha$ & 0.255 & 2798 & 0.228 \\
\hline $7.5005 \ldots \ldots \ldots \ldots \ldots$ & $\mathrm{Hu} \beta$ & 0.106 & 2278 & 0.095 \\
\hline 8.7577 ..................... & Н I $10-7$ & 0.049 & 3012 & 0.043 \\
\hline $11.3056 \ldots \ldots \ldots \ldots$ & Н I 9-7 & 0.092 & $\ldots$ & 0.081 \\
\hline $12.3685 \ldots \ldots \ldots \ldots$ & $\operatorname{Hu} \alpha$ & 0.190 & $\ldots$ & 0.166 \\
\hline $12.8101 \ldots \ldots \ldots \ldots$ & {$[\mathrm{Ne} \mathrm{II}]$} & 0.378 & 3451 & 0.330 \\
\hline \multicolumn{5}{|c|}{ Day $300^{c}$} \\
\hline $7.65 \ldots \ldots \ldots \ldots \ldots \ldots$ & {$[\mathrm{Ne} \mathrm{VI}]$} & $4.4 \pm 2.2$ & 3630 & $\ldots$ \\
\hline $14.32 \ldots \ldots \ldots \ldots \ldots \ldots$ & {$[\mathrm{Ne} \mathrm{v}]$} & $5.6 \pm 2.3$ & 3570 & $\cdots$ \\
\hline \multicolumn{5}{|c|}{ Day $334^{\mathrm{d}}$} \\
\hline 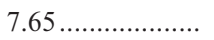 & {$[\mathrm{Ne} v \mathrm{VI}]$} & $3.5 \pm 1.9$ & 3278 & $\ldots$ \\
\hline $14.32 \ldots \ldots \ldots \ldots \ldots$ & {$[\mathrm{Ne} \mathrm{v}]$} & $7.9 \pm 2.8$ & 3493 & $\ldots$ \\
\hline $15.55 \ldots \ldots \ldots \ldots \ldots$ & {$[\mathrm{Ne} I I I]$} & $10.2 \pm 0.5$ & 4416 & $\ldots$ \\
\hline
\end{tabular}

a Observed $F_{\mathrm{Br} \alpha}=6.235 \times 10^{-11} \mathrm{ergs} \mathrm{cm}^{-2} \mathrm{~s}^{-1}$.

b Dereddened $F_{\mathrm{Br} \alpha}=7.190 \times 10^{-11} \mathrm{ergs} \mathrm{cm}^{-2} \mathrm{~s}^{-1}$.

${ }^{c}$ Fluxes not dereddened, $\times 10^{-12} \mathrm{ergs} \mathrm{cm}^{-2} \mathrm{~s}^{-1}$.

d Fluxes not dereddened, $\times 10^{-12} \mathrm{ergs} \mathrm{cm}^{-2} \mathrm{~s}^{-1}$.

(Shortridge 1993). We removed the strong $\mathrm{H}$ lines from the spectra of the standard stars before flux calibration. We report the fitted lines, fluxes, and widths in Table 3. An atmospheric correction also was applied to account for $\mathrm{CO}_{2}$ and $\mathrm{H}_{2} \mathrm{O}$ absorption bands. These regions were significantly more noisy, and therefore the measured line fluxes from these regions were omitted from the abundance analysis.

\section{3. $R G O$ Spectrograph}

Near contemporaneous optical spectra were obtained with the Royal Greenwich Observatory (RGO) Spectrograph on the AAT using the $\mathrm{f} / 8$ secondary and the $270 \mathrm{R}$ and 300B gratings, yielding spectral resolutions of $200<\lambda$ / $\Delta \lambda<400$. Again, both narrow- and wide-slit spectra were taken, respectively, for high $\mathrm{S} / \mathrm{N}$ and accurate flux calibration. For day 207.6, the narrow slit was 1".3 wide and the wide slit was 4".7 wide. For day 285.5, the narrow slit was 1 ."7 wide, while two wide slits (5" and 7".3) were used for both the nova and the calibrator. RGO Spectrograph data were reduced using the FIGARO data reduction system and standard techniques. To determine the line centers, integrated fluxes, and equivalent widths of the emission lines, the RGO Spectrograph nova spectra were fitted with model lines. When fitting emission lines, FIGARO offers the option of fitting either single-component or deblended Gaussians. The user may specify the number of Gaussian profiles to fit or leave it as a parameter. The results are reported in Table 4.

\subsection{Reddening Correction}

We determined the interstellar reddening using the Balmer decrement (Osterbrock 1989). Defining a "color excess" between $\mathrm{H} \alpha$ and $\mathrm{H} \beta$ :

$$
E(\mathrm{H} \alpha-\mathrm{H} \beta)=2.5 \log \left(\frac{[I(\mathrm{H} \alpha) / I(\mathrm{H} \beta)]_{\text {measured }}}{[I(\mathrm{H} \alpha) / I(\mathrm{H} \beta)]_{\text {intrinsic }}}\right),
$$

where $[I(\mathrm{H} \alpha) / I(\mathrm{H} \beta)]_{\text {measured }}$ is determined from our line fluxes and $[I(\mathrm{H} \alpha) / I(\mathrm{H} \beta)]_{\text {intrinsic }}=2.81$ for case $\mathrm{B}$, with an electron temperature of $T_{e}=10,000 \mathrm{~K}$ and electron density of $n_{e}=10^{6} \mathrm{~cm}^{-3}$ (Osterbrock 1989). We later show that these are reasonable temperature and density assumptions and note this is an iterative process. The relationship between this "color excess" and $E(B-V)$ results in (Howarth 1983)

$$
E(\mathrm{H} \alpha-\mathrm{H} \beta)=1.162 E(B-V) \operatorname{mag} .
$$


TABLE 3

IRIS NEAR-IR EMISSION-LINE DATA

\begin{tabular}{|c|c|c|c|c|c|c|c|c|c|c|}
\hline \multirow[b]{2}{*}{$\begin{array}{c}\lambda \\
(\mu \mathrm{m})\end{array}$} & \multirow[b]{2}{*}{ IDENTITY } & \multicolumn{3}{|c|}{ OBSERVED $F_{\lambda} / F_{\mathrm{Br} \gamma}$} & \multicolumn{3}{|c|}{ FWHM $\left(\mathrm{km} \mathrm{s}^{-1}\right)$} & \multicolumn{3}{|c|}{ DEREDDENED $F_{\lambda} / F_{\mathrm{Br} \gamma}$} \\
\hline & & $\begin{array}{l}\text { Day } \\
91.7^{\mathrm{a}}\end{array}$ & $\begin{array}{c}\text { Day } \\
179.6^{b}\end{array}$ & $\begin{array}{c}\text { Day } \\
266.4^{\mathrm{c}}\end{array}$ & $\begin{array}{l}\text { Day } \\
91.7\end{array}$ & $\begin{array}{c}\text { Day } \\
179.6\end{array}$ & $\begin{array}{c}\text { Day } \\
266.4\end{array}$ & $\begin{array}{l}\text { Day } \\
91.7^{\mathrm{d}}\end{array}$ & $\begin{array}{c}\text { Day } \\
179.6^{\mathrm{e}}\end{array}$ & $\begin{array}{c}\text { Day } \\
266.4^{f}\end{array}$ \\
\hline $0.8447 \ldots \ldots \ldots \ldots \ldots$ & $\mathrm{O}_{\mathrm{I}}$ & 0.365 & $\ldots$ & $\ldots$ & 3200 & $\ldots$ & $\ldots$ & 4.142 & $\ldots$ & $\ldots$ \\
\hline $0.9015 \ldots \ldots \ldots \ldots . . . .$. & $\mathrm{Pa} 10-3$ & 0.185 & $\ldots$ & $\ldots$ & 3141 & $\ldots$ & $\ldots$ & 1.559 & $\ldots$ & $\ldots$ \\
\hline $0.9229 \ldots \ldots \ldots \ldots \ldots$ & $\mathrm{Pa} 9-3$ & 0.240 & $\ldots$ & $\ldots$ & 3191 & $\ldots$ & $\ldots$ & 1.831 & $\ldots$ & $\ldots$ \\
\hline $0.9345 \ldots \ldots \ldots \ldots \ldots$ & He II $8-5$ & 0.287 & $\ldots$ & $\ldots$ & $\ldots$ & $\ldots$ & $\ldots$ & 2.082 & $\ldots$ & $\ldots$ \\
\hline $0.9546 \ldots \ldots \ldots \ldots$ & $\mathrm{Pa} \epsilon+[\mathrm{Si} \mathrm{III}]$ & 0.315 & $\ldots$ & 5.280 & 3520 & $\ldots$ & $\ldots$ & 2.092 & $\ldots$ & 34.990 \\
\hline $0.9900 \ldots \ldots \ldots \ldots . .$. & $?$ & 0.086 & $\ldots$ & $\ldots$ & $\ldots$ & $\ldots$ & $\ldots$ & 0.495 & $\ldots$ & $\ldots$ \\
\hline 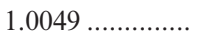 & $\operatorname{Pa} \delta$ & 0.903 & 1.543 & 2.546 & 3181 & 3500 & 2983 & 4.895 & 8.371 & 13.810 \\
\hline $1.0123 \ldots \ldots \ldots \ldots . . .$. & He II 5-4 & 0.138 & 0.415 & 0.926 & 2843 & 3627 & 2369 & 0.728 & 2.188 & 4.886 \\
\hline $1.0420 \ldots \ldots \ldots \ldots$ & He II 13-6 & 0.067 & 0.429 & 0.912 & $\ldots$ & $\ldots$ & $\ldots$ & 0.316 & 2.036 & 4.327 \\
\hline $1.0830 \ldots \ldots \ldots \ldots . . . .$. & He I & 8.380 & 19.233 & 22.883 & 3360 & 3217 & 3322 & 34.728 & 79.688 & 94.824 \\
\hline 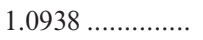 & $\operatorname{Pa} \gamma$ & 1.498 & 0.395 & 2.046 & 3350 & 3624 & 3672 & 6.004 & 1.583 & 8.199 \\
\hline $1.1287 \ldots \ldots \ldots \ldots . .$. & $\mathrm{O}_{\mathrm{I}}$ & 0.428 & 0.815 & $\ldots$ & 3586 & $\ldots$ & $\ldots$ & 1.548 & 2.946 & $\ldots$ \\
\hline $1.1673 \ldots \ldots \ldots \ldots . .$. & He II blend & 0.360 & 0.266 & 1.686 & $\ldots$ & $\ldots$ & 3081 & 1.172 & 0.866 & 5.487 \\
\hline $1.1880 \ldots \ldots \ldots \ldots . .$. & $\mathrm{He} \mathrm{I}+[\mathrm{Fe} \mathrm{II}]$ & 0.095 & 0.540 & 1.254 & $\ldots$ & $\ldots$ & $\ldots$ & 0.293 & 1.667 & 3.872 \\
\hline $1.2530 \ldots \ldots \ldots \ldots \ldots$ & He I & 0.062 & $\ldots$ & $\ldots$ & $\ldots$ & $\ldots$ & $\ldots$ & 0.164 & $\ldots$ & $\ldots$ \\
\hline $1.2820 \ldots \ldots \ldots \ldots . .$. & $\mathrm{Pa} \beta+\mathrm{He}$ & 0.633 & 3.479 & 6.089 & 3391 & 3624 & 3625 & 1.579 & 8.683 & 15.197 \\
\hline $1.2990 \ldots \ldots \ldots \ldots . .$. & He I & 0.014 & $\ldots$ & $\ldots$ & $\ldots$ & $\ldots$ & $\ldots$ & 0.034 & $\ldots$ & $\ldots$ \\
\hline $1.3160 \ldots \ldots \ldots \ldots . .$. & $\mathrm{O}_{\mathrm{I}}$ & 0.046 & $\ldots$ & $\ldots$ & $\ldots$ & $\ldots$ & $\ldots$ & 0.107 & $\ldots$ & $\ldots$ \\
\hline 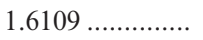 & Br 13-4 & 0.182 & 0.173 & $\ldots$ & 3349 & 3722 & $\ldots$ & 0.275 & 0.261 & $\ldots$ \\
\hline $1.6407 \ldots \ldots \ldots \ldots . .$. & $\operatorname{Br} 12-4$ & 0.228 & 0.218 & $\ldots$ & 3380 & 3654 & $\ldots$ & 0.335 & 0.319 & $\ldots$ \\
\hline $1.6806 \ldots \ldots \ldots \ldots . .$. & Br 11-4 & 0.316 & 0.205 & 0.191 & 3247 & 3746 & 2675 & 0.445 & 0.288 & 0.269 \\
\hline $1.6920 \ldots \ldots \ldots \ldots . . . .$. & He II $12-7$ & $\ldots$ & 0.121 & 0.075 & $\ldots$ & $\ldots$ & $\ldots$ & $\ldots$ & 0.168 & 0.104 \\
\hline $1.7002 \ldots \ldots \ldots \ldots . .$. & He I & 0.190 & & $\ldots$ & 3279 & $\ldots$ & $\ldots$ & 0.263 & $\ldots$ & $\ldots$ \\
\hline $1.7160 \ldots \ldots \ldots \ldots . .$. & $?$ & $\ldots$ & 0.235 & $\ldots$ & $\ldots$ & $\ldots$ & $\ldots$ & $\ldots$ & 0.319 & $\ldots$ \\
\hline $1.7362 \ldots \ldots \ldots \ldots . . .$. & Br 10-4 & 0.540 & 0.683 & 0.655 & 3453 & 3194 & 3453 & 0.721 & 0.911 & 0.874 \\
\hline $1.9640 \ldots \ldots \ldots \ldots . .$. & [Si vI] & $\ldots$ & 6.258 & 2.108 & $\ldots$ & $\ldots$ & $\ldots$ & $\ldots$ & 7.009 & 2.360 \\
\hline $2.0400 \ldots \ldots \ldots \ldots \ldots$ & [A1 IX] & $\ldots$ & 0.180 & 0.115 & $\ldots$ & 3512 & $\ldots$ & $\ldots$ & 0.192 & 0.123 \\
\hline $2.0581 \ldots \ldots \ldots \ldots . .$. & He I & 1.008 & 1.124 & 0.654 & 3277 & 3350 & 3453 & 1.067 & 1.190 & 0.692 \\
\hline $2.0900 \ldots \ldots \ldots \ldots . . .$. & $?$ & $\ldots$ & $\ldots$ & 0.036 & $\ldots$ & $\ldots$ & $\ldots$ & $\ldots$ & $\ldots$ & 0.038 \\
\hline $2.1126 \ldots \ldots \ldots \ldots$ & He I & 0.371 & 0.687 & 0.685 & $\ldots$ & $\ldots$ & $\ldots$ & 0.381 & 0.705 & 0.704 \\
\hline $2.1655 \ldots \ldots \ldots \ldots \ldots$ & $\mathrm{Br} \gamma$ & 1.000 & 1.000 & 1.000 & 3461 & 3253 & 3461 & 1.000 & 1.000 & 1.000 \\
\hline $2.1885 \ldots \ldots \ldots \ldots \ldots$ & He II 10-7 & 0.321 & 0.137 & 0.207 & 2877 & 3000 & $\ldots$ & 0.318 & 0.136 & 0.205 \\
\hline $2.2190 \ldots \ldots \ldots \ldots \ldots$ & $?$ & 0.031 & $\ldots$ & $\ldots$ & $\ldots$ & $\ldots$ & $\ldots$ & 0.030 & $\ldots$ & $\ldots$ \\
\hline $2.3205 \ldots \ldots \ldots \ldots \ldots$ & [Ca VIII] & $\ldots$ & 0.318 & 0.065 & $\ldots$ & $\ldots$ & $\ldots$ & $\ldots$ & 0.297 & 0.060 \\
\hline $2.4827 \ldots \ldots \ldots \ldots \ldots$ & [Si VII] & $\ldots$ & 6.368 & 0.701 & $\ldots$ & $\ldots$ & $\ldots$ & $\ldots$ & 5.603 & 0.617 \\
\hline
\end{tabular}

Using data obtained on day 207.6, we find $E(B-V)=$ $1.76 \pm 0.1 \mathrm{mag}$, and for day 285.5, we find $E(B-V)=$ $1.95 \pm 0.1 \mathrm{mag}$. Using these values and a standard interstellar reddening law (Rieke \& Lebofsky 1985), we correct our measured line fluxes for extinction. For our IR spectra, we take $E(B-V)=1.9 \pm 0.1 \mathrm{mag}$ as a representative value and similarly correct line fluxes for extinction. As IR measurements are less affected by interstellar extinction than are optical measurements, the corrections thus determined are sufficiently accurate for our analysis. Our derived value of $E(B-V) \simeq 1.90 \mathrm{mag}$ corresponds to an $A_{V}=5.9 \pm 0.3$. This result agrees well with the NASA/IPAC Extragalactic Database (NED) estimate of $E(B-V)=1.99 \mathrm{mag}$ for this position on the sky. However, because $\mathrm{CP}$ Cru is located in the Galactic plane, we note that general $E(B-V)$ estimates, such as those from NED, must be used only for comparison. Both the observed and dereddened line fluxes are reported in Tables 2, 3, and 4.

\section{RESULTS AND DISCUSSION}

\subsection{Expansion Distance and $M_{V}$}

Downes \& Duerbeck (2000) determined a distance to CP Cru based on HST images of the expanding nova shell and low-resolution spectra yielding expansion velocities, $v_{\exp } \simeq$ $2000 \mathrm{~km} \mathrm{~s}^{-1}$ (Della Valle \& Benetti 1997). ISO SWS spectra from day 24.7 show nearly rectangular line profiles such that the FWHM velocity is only slightly less than the full-width zero-intensity (FWZI) velocity (Fig. 2). Indeed, for [Ne II] $12.8 \mu \mathrm{m}, \mathrm{FWZI}=3560 \mathrm{~km} \mathrm{~s}^{-1}$ and FWHM $=3060 \mathrm{~km} \mathrm{~s}^{-1}$. The radial expansion velocity in this case is then half of the full-width velocities. We find for [Ne II] $12.8 \mu \mathrm{m}: v_{\exp }=1780$ and $1530 \mathrm{~km} \mathrm{~s}^{-1}$ from the FWZI and FWHM, respectively. Because the Gaussian FWHM is less subjective than a determination of where the line profile meets the continuum (FWZI), we contend that the FWHM is the more accurate velocity. We determine $v_{\mathrm{FWHM}}$ for each date of observation 
TABLE 4

RGO Spectrograph Optical Emission-Line Data

\begin{tabular}{|c|c|c|c|c|c|c|c|}
\hline \multirow{2}{*}{$\begin{array}{c}\lambda \\
(\mu \mathrm{m})\end{array}$} & \multirow[b]{2}{*}{ IDENTITY } & \multicolumn{2}{|c|}{ OBSERVED $F_{\lambda} / F_{\mathrm{H} \beta}$} & \multicolumn{2}{|c|}{ FWHM $\left(\mathrm{km} \mathrm{s}^{-1}\right)$} & \multicolumn{2}{|c|}{ DEREDDENED $F_{\lambda} / F_{\mathrm{H} \beta}$} \\
\hline & & Day $207.6^{\mathrm{a}}$ & Day $285.5^{\mathrm{b}}$ & Day 207.6 & Day 285.5 & Day $207.6^{c}$ & Day $285.5^{\mathrm{d}}$ \\
\hline $0.3345 \ldots \ldots \ldots \ldots \ldots$ & {$[\mathrm{Ne} \mathrm{v}]$} & . & 1.003 & $\ldots$ & . & $\ldots$ & 10.620 \\
\hline $0.3426 \ldots \ldots \ldots \ldots \ldots$ & {$[\mathrm{Ne} \mathrm{v}]$} & 3.561 & 2.788 & 4910 & 4078 & 25.500 & 24.690 \\
\hline $0.3727 \ldots \ldots \ldots \ldots . .$. & [O II] & 0.273 & 0.432 & $\ldots$ & $\ldots$ & 1.235 & 2.298 \\
\hline 0.3869 ................. & {$[\mathrm{Ne} \mathrm{III}]$} & 4.392 & 3.414 & 4128 & 3671 & 17.030 & 15.320 \\
\hline $0.3968 \ldots \ldots \ldots \ldots \ldots$ & {$[\mathrm{Ne} \mathrm{III}]$} & 1.507 & 0.980 & 2678 & 3356 & 5.195 & 3.863 \\
\hline $0.4101 \ldots \ldots \ldots \ldots . .$. & $\mathrm{H} \delta+[\mathrm{S} \mathrm{II}]$ & 0.496 & 0.477 & $\ldots$ & $\ldots$ & 1.443 & 1.558 \\
\hline $0.4340 \ldots \ldots \ldots \ldots \ldots$ & $\mathrm{H} \gamma$ & 0.291 & 0.441 & & 2845 & 0.612 & 1.007 \\
\hline $0.4363 \ldots \ldots \ldots \ldots .$. & [O III] & 1.853 & 1.004 & 2698 & 3184 & 3.783 & 2.214 \\
\hline $0.4640 \ldots \ldots \ldots \ldots \ldots$ & N III & 0.232 & 0.736 & 2312 & 3766 & 0.319 & 1.048 \\
\hline $0.4686 \ldots \ldots \ldots \ldots \ldots$ & He II & 0.650 & 0.523 & 3850 & 2969 & 0.837 & 0.693 \\
\hline $0.4720 \ldots \ldots \ldots \ldots \ldots$ & {$[\mathrm{Ne}$ IV $]$} & 0.978 & 0.249 & 2687 & 1998 & 1.200 & 0.312 \\
\hline $0.4861 \ldots \ldots \ldots \ldots \ldots$ & $\mathrm{H} \beta$ & 1.000 & 1.000 & 3051 & 3330 & 1.000 & 1.000 \\
\hline $0.4959 \ldots \ldots \ldots \ldots . . .$. & [O III $]$ & 4.397 & 15.850 & 3721 & 3774 & 3.817 & 13.550 \\
\hline $0.5007 \ldots \ldots \ldots \ldots \ldots$ & [O III] & 30.420 & 41.240 & 3300 & 3353 & 24.640 & 32.650 \\
\hline $0.5721 \ldots \ldots \ldots \ldots \ldots$ & {$[\mathrm{Fe}$ VII $]$} & 0.802 & 0.538 & $\ldots$ & $\ldots$ & 0.264 & 0.157 \\
\hline $0.5755 \ldots \ldots \ldots \ldots \ldots$ & {$[\mathrm{N}$ II $]$} & 2.146 & 1.728 & 3021 & 3142 & 0.683 & 0.486 \\
\hline $0.5876 \ldots \ldots \ldots \ldots \ldots$ & He I & 0.584 & 0.568 & 4084 & 3228 & 0.165 & 0.140 \\
\hline $0.6087 \ldots \ldots \ldots \ldots . .$. & {$[\mathrm{Fe}$ VII $]$} & 0.587 & 0.567 & 2904 & 3148 & 0.136 & 0.112 \\
\hline $0.6300 \ldots \ldots \ldots \ldots \ldots$ & {$[\mathrm{O}$ I] } & 0.514 & 0.207 & 3252 & $\ldots$ & 0.098 & 0.033 \\
\hline $0.6363 \ldots \ldots \ldots \ldots$ & [O I $]$ & 0.344 & 0.160 & 3300 & $\ldots$ & 0.062 & 0.024 \\
\hline $0.6548 \ldots \ldots \ldots \ldots \ldots$ & {$[\mathrm{N}$ II] } & 1.131 & 0.509 & 790 & 670 & 0.174 & 0.064 \\
\hline $0.6563 \ldots \ldots \ldots \ldots . .$. & $\mathrm{H} \alpha$ & 18.490 & 22.660 & 3802 & 2993 & 2.810 & 2.809 \\
\hline $0.6583 \ldots \ldots \ldots \ldots \ldots$ & {$\left[\mathrm{N}_{\mathrm{II}}\right]$} & 1.547 & 2.195 & 780 & 900 & 0.231 & 0.267 \\
\hline $0.6678 \ldots \ldots \ldots \ldots . .$. & $\mathrm{He}_{\mathrm{I}}$ & 0.167 & 0.206 & 2234 & $\ldots$ & 0.023 & 0.023 \\
\hline $0.7005 \ldots \ldots \ldots \ldots . . .$. & {$[\mathrm{Ar} v]$} & 0.131 & 0.226 & 2000 & 3835 & 0.014 & 0.019 \\
\hline $0.7065 \ldots \ldots \ldots \ldots \ldots$ & $\mathrm{He}_{\mathrm{I}}$ & 0.294 & 0.163 & 2500 & 3156 & 0.030 & 0.013 \\
\hline $0.7136 \ldots \ldots \ldots \ldots \ldots$ & [Ar III] & 0.793 & 0.954 & 3621 & 3597 & 0.077 & 0.072 \\
\hline $0.7325 \ldots \ldots \ldots \ldots \ldots$ & [O II] blend & 7.046 & 5.967 & $\ldots$ & $\ldots$ & 0.598 & 0.388 \\
\hline $0.7599 \ldots \ldots \ldots \ldots . . .$. & $?$ & 0.738 & 0.226 & $\ldots$ & $\ldots$ & 0.019 & 0.012 \\
\hline $0.7734 \ldots \ldots \ldots \ldots . .$. & [Ar III]? & 0.973 & 0.746 & $\ldots$ & $\ldots$ & 0.063 & 0.036 \\
\hline $0.8252 \ldots \ldots \ldots \ldots \ldots$ & $?$ & 1.628 & 1.420 & $\ldots$ & $\ldots$ & 0.078 & 0.049 \\
\hline $0.9069 \ldots \ldots \ldots \ldots . . .$. & {$[\mathrm{S}$ III $]$} & $\ldots$ & 2.398 & $\ldots$ & 3664 & $\ldots$ & 0.053 \\
\hline
\end{tabular}

a Observed $F_{\mathrm{H} \beta}$ (day 207.6) $=1.828 \times 10^{-14} \mathrm{ergs} \mathrm{cm}^{-2} \mathrm{~s}^{-1}$.

${ }^{\mathrm{b}}$ Observed $F_{\mathrm{H} \beta}$ (day 285.5) $=8.204 \times 10^{-15} \mathrm{ergs} \mathrm{cm}^{-2} \mathrm{~s}^{-1}$.

c Dereddened $F_{\mathrm{H} \beta}\left(\right.$ day 207.6) $=6.577 \times 10^{-12} \mathrm{ergs} \mathrm{cm}^{-2} \mathrm{~s}^{-1}$

${ }^{\mathrm{d}}$ Dereddened $F_{\mathrm{H} \beta}\left(\right.$ day 285.5) $=5.572 \times 10^{-12} \mathrm{ergs} \mathrm{cm}^{-2} \mathrm{~s}^{-1}$.

by taking the simple average of the Gaussian FWHM determined from the line fits. Because the $v_{\text {FWHM }}$ could be interpreted as constant throughout our observations, we determined an average $v_{\mathrm{FWHM}}$ for all observations as the weighted average of velocities determined from each spectrum.

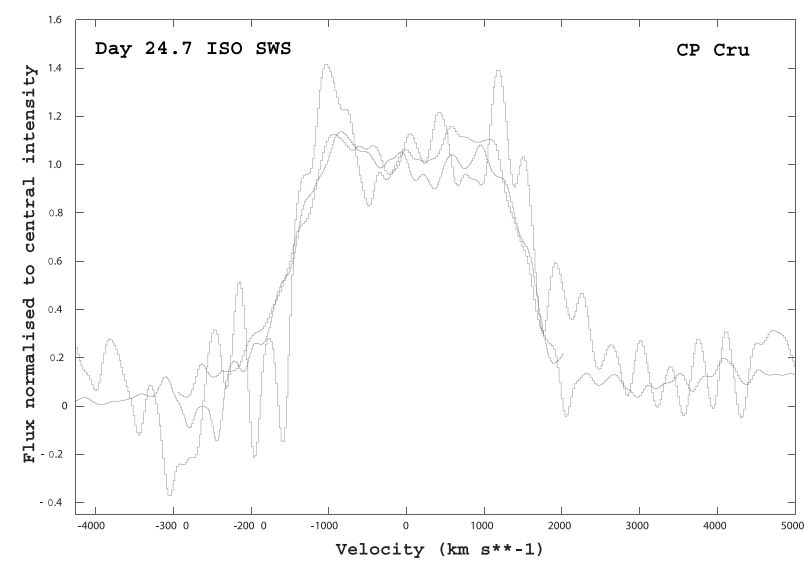

FIG. 2.-Nearly rectangular and double-peaked velocity profiles of [Ne II] $12.8 \mu \mathrm{m}, \mathrm{Br} \alpha$, and $\mathrm{Br} \beta$ from day 24.7 ISO SWS spectrum.
We find the average $v_{\mathrm{FWHM}}=3300 \pm 100 \mathrm{~km} \mathrm{~s}^{-1}$, and therefore, $v_{\text {exp }}=1650 \pm 100 \mathrm{~km} \mathrm{~s}^{-1}$. Using the expansion parallax, this gives a distance to CP Cru of $2.6 \mathrm{kpc}$. The velocity dispersion implies an error of $0.2 \mathrm{kpc}$; however, this distance determination assumes $v_{\text {exp }}$ is spherically symmetric, which can introduce errors of $15 \%$ (Ford \& Ciardullo 1988). Therefore, the error on the distance is approximately $0.5 \mathrm{kpc}$. Furthermore, using this distance and our average extinction, $A_{V}=5.9 \pm 0.3$, we determine $M_{V}=-8.7 \pm 0.5$. This measurement corresponds to the maximum observed brightness on day 0.96 of $m_{V}=9.25$ (Harris et al. 1999; Liller et al. 1996). As an independent comparison, we can use the maximum magnitude versus rate of decline (MMRD) relationship of Della Valle \& Livio (1995) to determine $M_{V}$ and distance. Using $t_{2}=4.3$ days from Figure 1, we find $M_{V}=-8.9 \pm 0.5 \mathrm{mag}$. This corresponds to a distance of $2.8 \pm 0.5 \mathrm{kpc}$, in agreement with the expansion parallax. Finally, the photoionization model analysis $(\S 4)$ indicates a maximum distance on the order of $2.7 \mathrm{kpc}$.

\subsection{Emission Lines}

Our day 24.7 ISO SWS spectrum is shown in Figure 3, and the observed lines are listed in Table 2. At this 


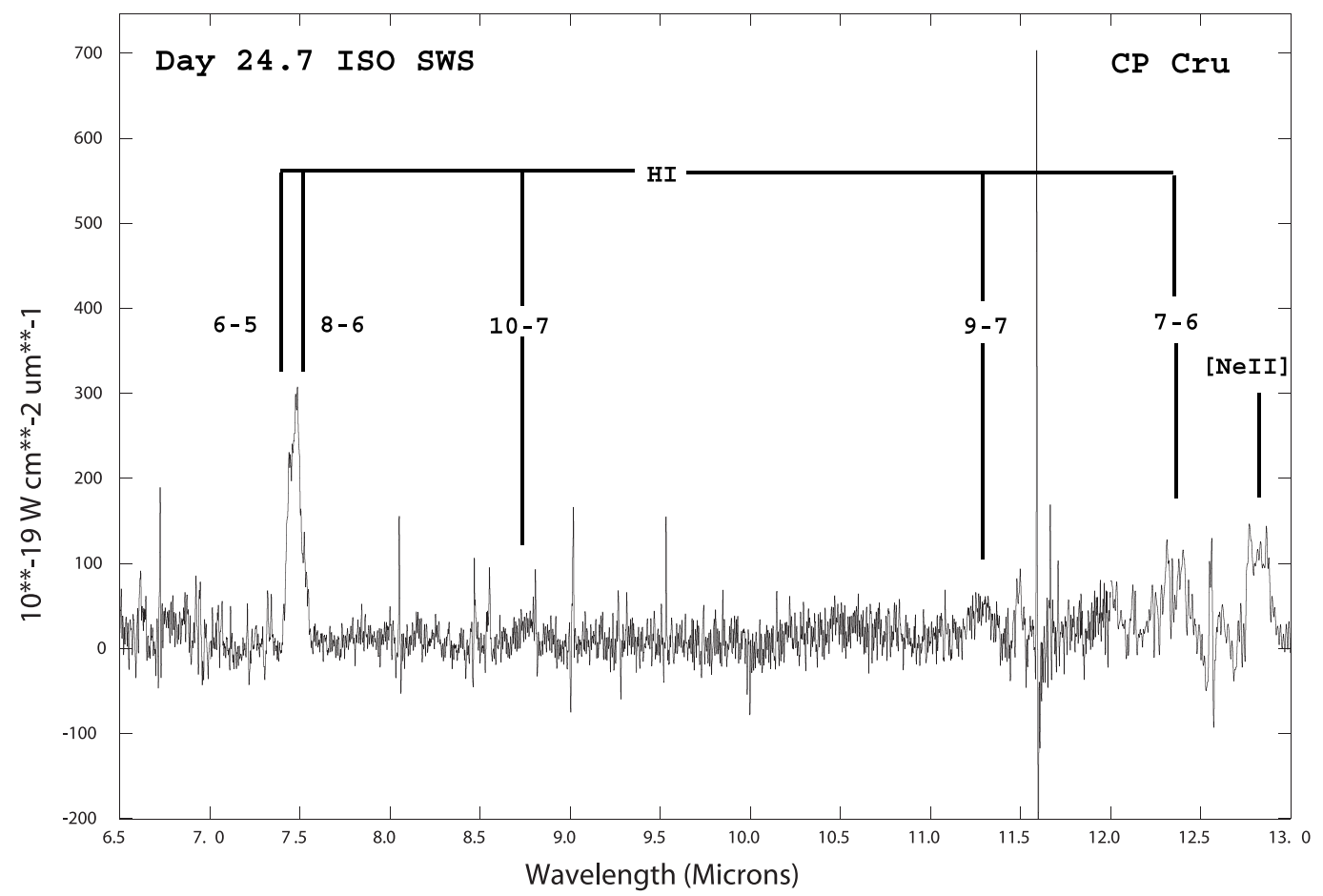

FIG. 3.-Sample spectrum from ISO SWS day 24.7 showing 6.4-13.0 $\mu \mathrm{m}$. Note the strong H I lines and the double-peaked [Ne II] $12.8 \mu \mathrm{m}$ line.

epoch, $\mathrm{H}$ I lines such as $\operatorname{Br} \alpha, \operatorname{Br} \beta$, and the Pfund series lines at $3.30,3.74,4.65$, and $7.46 \mu \mathrm{m}$ dominate the spectrum, in addition to a strong [Ne II] $12.81 \mu \mathrm{m}$ line. This is the earliest onset of [Ne II] emission recorded in a nova. At this early epoch, $v_{\mathrm{FWHM}}=3100 \pm 300 \mathrm{~km} \mathrm{~s}^{-1}$. In the IRIS spectrum of day 91.7 (Fig. 4; Table 3), the
He I triplet at $1.08 \mu \mathrm{m}$ is the strongest line; however, the strong $\mathrm{H}$ I lines such as $\mathrm{Br} \gamma$ and $\mathrm{Pa} \beta$ and the [O I] triplet at $1.13 \mu \mathrm{m}$ are also pronounced. In addition, the measured expansion velocity on day $91.7\left(v_{\mathrm{FWHM}}=\right.$ $3300 \pm 200 \mathrm{~km} \mathrm{~s}^{-1}$ ) is comparable to that determined on day 24.7 .

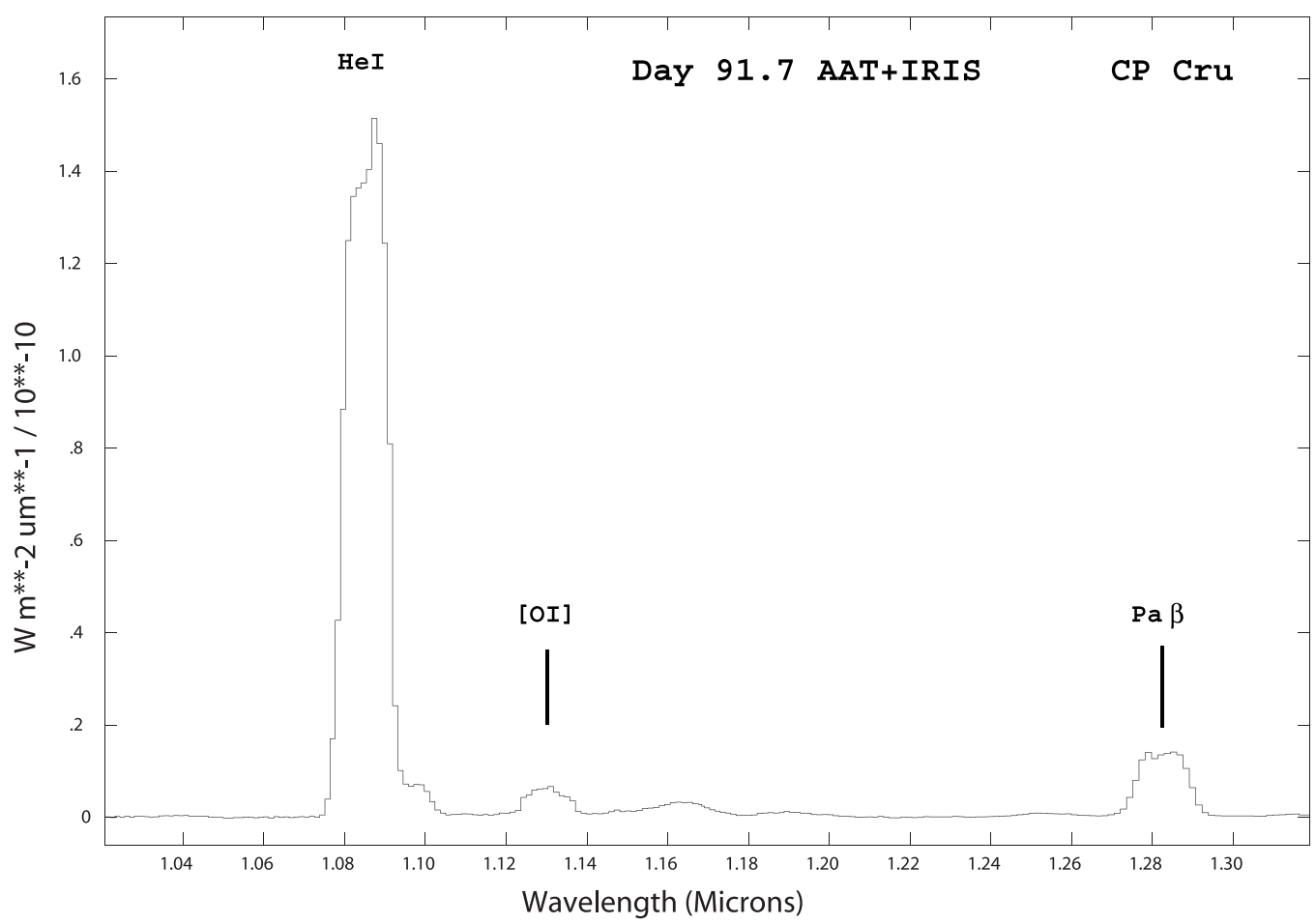

FIG. 4.-Sample spectrum from AAT IRIS day 91.7 showing 1.0-1.5 $\mu \mathrm{m}$. The He I triplet at $1.08 \mu \mathrm{m}$ is the strongest line observed on this date. 


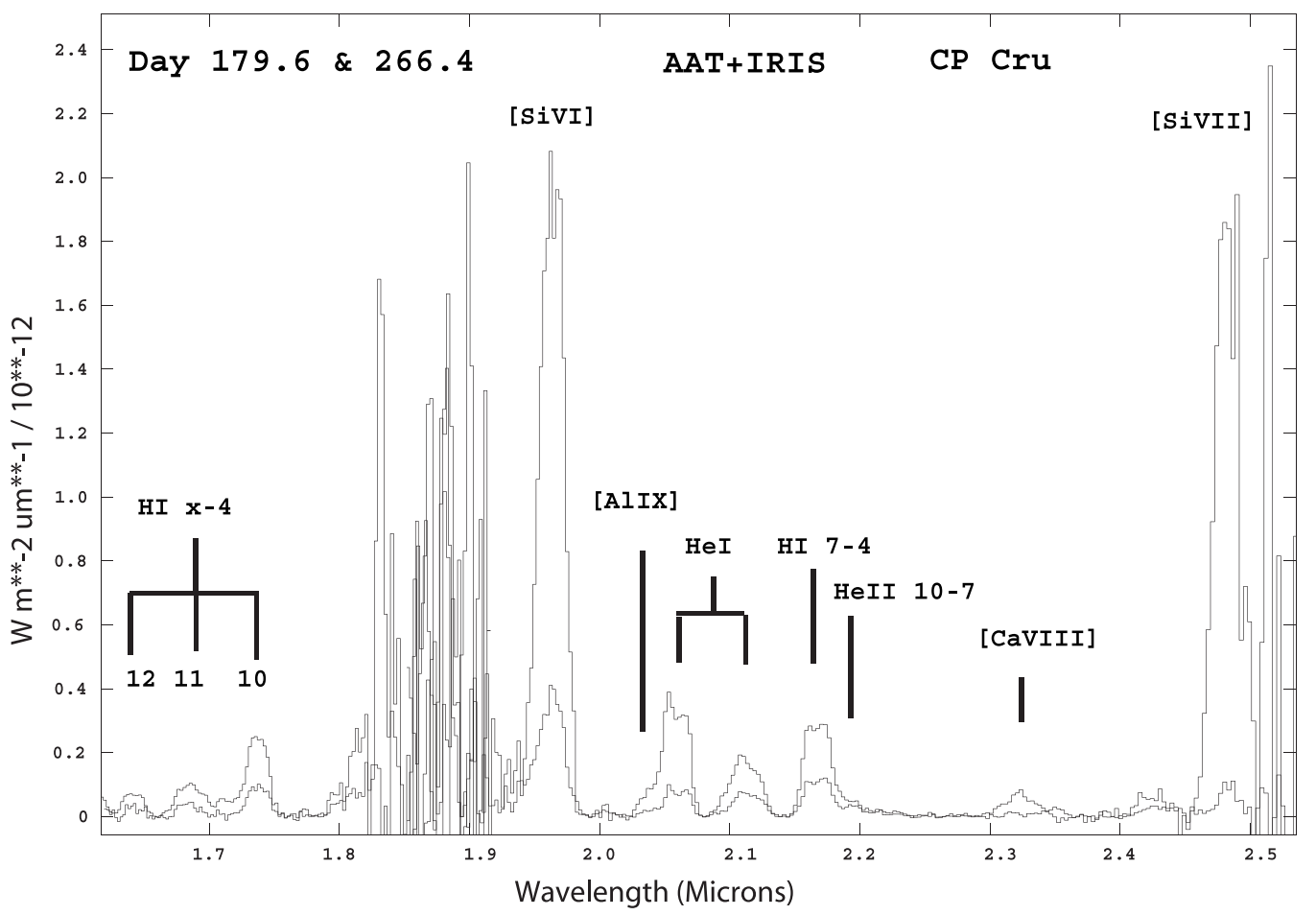

FIG. 5.-AAT IRIS days 179.6 (top spectrum) and 266.7 (bottom spectrum) spectra from 1.7 to $2.5 \mu \mathrm{m}$. Note the prominent " coronal" lines [Al Ix], [Si vI], [Si VII], and [Ca VIII].

The IRIS spectrum of day 179.6 (Fig. 5; Table 3) shows lower integrated fluxes in emission lines than that of day 91.7. The $\mathrm{Br} \gamma, \operatorname{Pa} \beta$, and the $\mathrm{He}$ I triplet at $1.08 \mu \mathrm{m}$ are still prominent. Moreover, highly ionized "coronal" lines such as [Si VI] 1.96, [Si VII] 2.48, and [Ca VIII] $2.32 \mu \mathrm{m}$ are now evident at this epoch. Additionally, there is a "shoulder" on the He I $2.06 \mu \mathrm{m}$ line that we attribute to [A1 Ix] $2.04 \mu \mathrm{m}$. On day $179.6, v_{\text {FWHM }}=3500 \pm 200 \mathrm{~km} \mathrm{~s}^{-1}$.

Our first optical spectrum was taken on day 207.6 and is shown in Figures 6 and 7. This spectrum is dominated by the nebular lines of [O III] $\lambda \lambda 4363,4959$, and 5007, [Ne III] $\lambda 3869,[\mathrm{Ne} v] \lambda 3426$, and the Balmer series. There are also

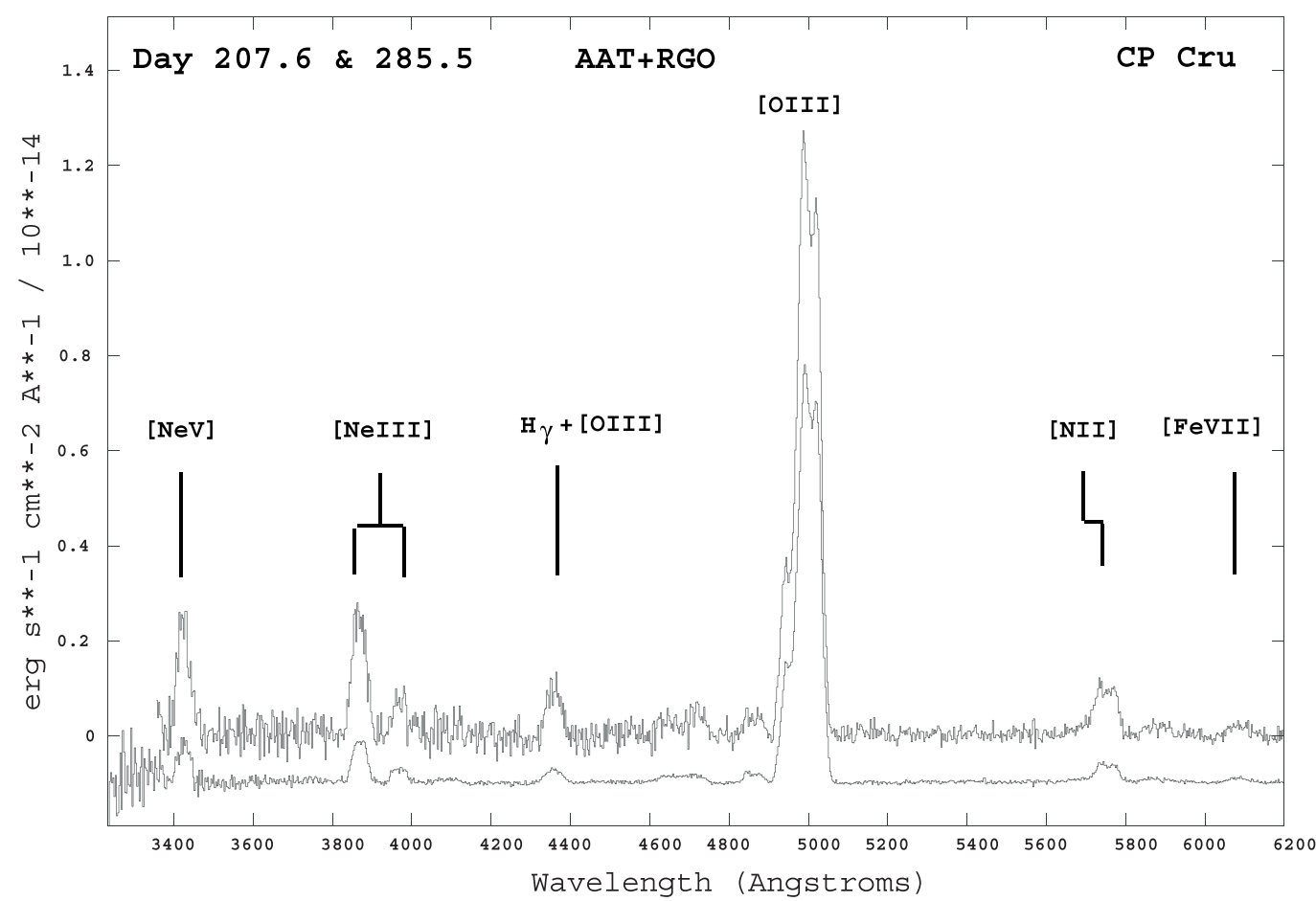

FIG. 6.-AAT RGO Spectrograph days 207.6 (top spectrum) and 285.5 (bottom spectrum) spectra from $\lambda \lambda 3400$ to 6200 showing typical nebular lines 


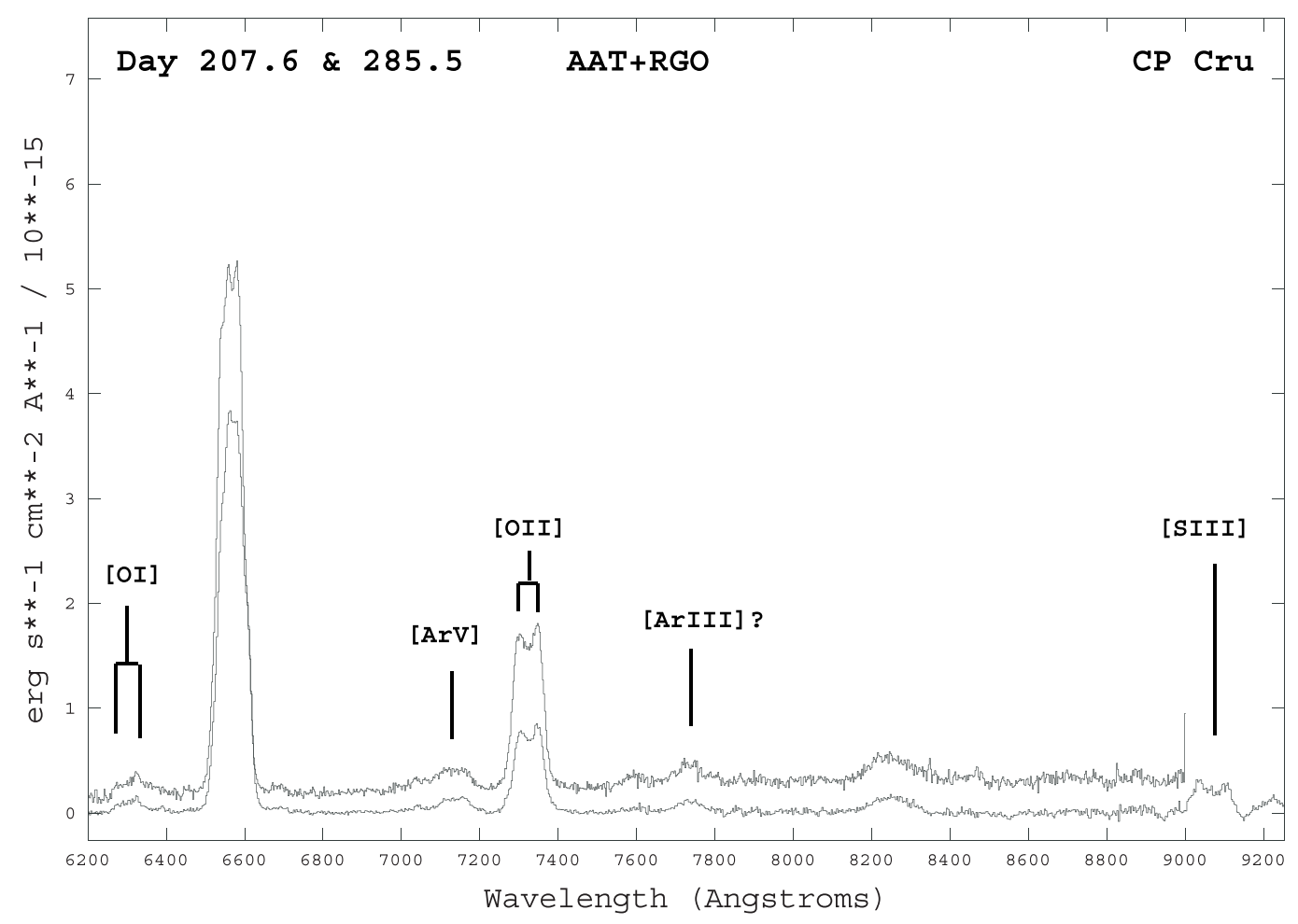

FIG. 7.-AAT RGO Spectrograph days 207.6 (top spectrum) and 285.5 (bottom spectrum) spectra from 6200 to $9200 \AA$

lines due to [Ar III] $\lambda 7136,[\mathrm{Ar} v] \lambda 7005,[\mathrm{~N}$ II] $\lambda \lambda 5755,6548$, and 6583, [O II] $\lambda \lambda 3727$ and 7325, and [Fe VII] $\lambda \lambda 5721$ and 6086. Line fluxes and velocities are presented in Table 4 . We find $v_{\mathrm{FWHM}}=3200 \pm 700 \mathrm{~km} \mathrm{~s}^{-1}$. We did not include the expansion velocities of [N II] $\lambda \lambda 6548$ and 6583 in the average because of their much slower velocities $\left(v_{\mathrm{FWHM}}<800 \mathrm{~km}\right.$ $\mathrm{s}^{-1}$ ). The much lower velocities suggest that the $\mathrm{N}$ lines formed farther inside the ejecta (see the density discussion as it relates to shell thickness $\S 4.4$ ). The slower expansion also means that the wings of $\mathrm{H} \alpha$ overlap [N II] $\lambda \lambda 6548$ and 6583 , necessitating the inclusion of three components in the fit to $\mathrm{H} \alpha$ (see $\S 2.3$ ). Yet, based on the strength of [N II] $\lambda 5755$ and canonical emissivities (Shaw \& Dufour 1995), we assert that $\lambda \lambda 6548$ and 6583 contribute to the measured flux.

Our third IRIS spectrum on day 266.4 (Fig. 5 and Table 3) was taken with a narrow slit, so we report only relative fluxes. While $\mathrm{He}$ I continues to be the strongest line, $\mathrm{Pa} \delta$ at $1.0049 \mu \mathrm{m}$ and a $\mathrm{H} / \mathrm{He}$ blend at $1.282 \mu \mathrm{m}$ are stronger with respect to $\mathrm{Br} \gamma$. More notable, however, is the fading of the "coronal" lines of [Si VI] 1.96, [Ca VIII] 2.32, and [Si VII] $2.48 \mu \mathrm{m}$ when compared with day 179.6. Additionally, the "shoulder" on the He I $2.06 \mu \mathrm{m}$ line attributed to [Al Ix] $2.04 \mu \mathrm{m}$ was not detected. The average $v_{\mathrm{FWHM}}=3200 \pm$ $400 \mathrm{~km} \mathrm{~s}^{-1}$.

The RGO Spectrograph spectrum on day 285.5 shows development consistent with a decrease in the shell density due to expansion when compared with the day 207.6 spectrum (see Figs. 6 and $7 ; \S 4.4$ ). First, the $[\mathrm{O}$ III $] \lambda \lambda 4959+5007$ doublet has greatly increased in flux with respect to $\mathrm{H} \beta$, while [O III] $\lambda 4363$ is weaker. Lines due to [Ne III] $\lambda 3869$ and $[\mathrm{Ne} \mathrm{v}] \lambda 3426$ are also weaker as are [O I] $\lambda \lambda 6300$ and 6363. In both optical observations, the flux ratio of $\left[\mathrm{O}_{\mathrm{I}}\right] \lambda 6300 /$ $\lambda 6363$ is about 1.5 versus the optically thin value of 3 . We attribute this discrepancy to the possibility that the $[\mathrm{O} \mathrm{I}]$ is shielded from high-energy photons in the denser clumps of material in the ejecta and may not be completely optically thin. We note that $\left[\mathrm{O}\right.$ II] $\lambda 3727$ is stronger, but $\left[\mathrm{O}_{\mathrm{II}}\right] \lambda 7325$ is weaker. The fluxes and velocities of all observed lines are presented in Table 4 . The average expansion velocity was $v_{\text {FWHM }}=3300 \pm 500 \mathrm{~km} \mathrm{~s}^{-1}$.

Our final observations are spectra obtained on days 300 and 334 post outburst using ISO SWS (see Figs. 8 and 9). CP Cru had faded in the IR at this epoch and, as a result, only two lines are readily detectable in both spectra: [Ne VI] 7.65 and [Ne v] $14.325 \mu \mathrm{m}$. The line fluxes remained constant between the two sets of ISO observations, which is expected due to the relatively small time between observations. We also detected [Ne III] $15.555 \mu \mathrm{m}$ on day 334, but we could not fit the same line on day 300 because of a noise spike at that wavelength. We report the line fits in Table 2.

As described in Paper I for V1425 Aql, we attribute the higher ionization states to the expansion of the ejecta while the effective temperature of the white dwarf (WD) increased, driving ionization to higher states (Gehrz et al. 1998). Observations of the neon nova V1974 Cyg show that the decline of [Ne II] emission is correlated with the rise of [Ne VI] emission (Hayward et al. 1996). However, weaker [Ne II] emission recovered past day 800 in that nova. Notably absent in the ISO SWS spectra of CP Cru is the [O IV] $25.89 \mu \mathrm{m}$ line, which was strong and temporally persistent for many hundreds of days in V1974 Cyg (Salama et al. 1996), V705 Cas (Salama et al. 1999), and V1425 Aql (Paper I).

\subsection{Temperature and Density Determination}

Due to the greater wavelength coverage afforded by near contemporaneous RGO Spectrograph (optical, day 285.5) and ISO SWS (IR, day 300.4) spectra, an estimate of the 


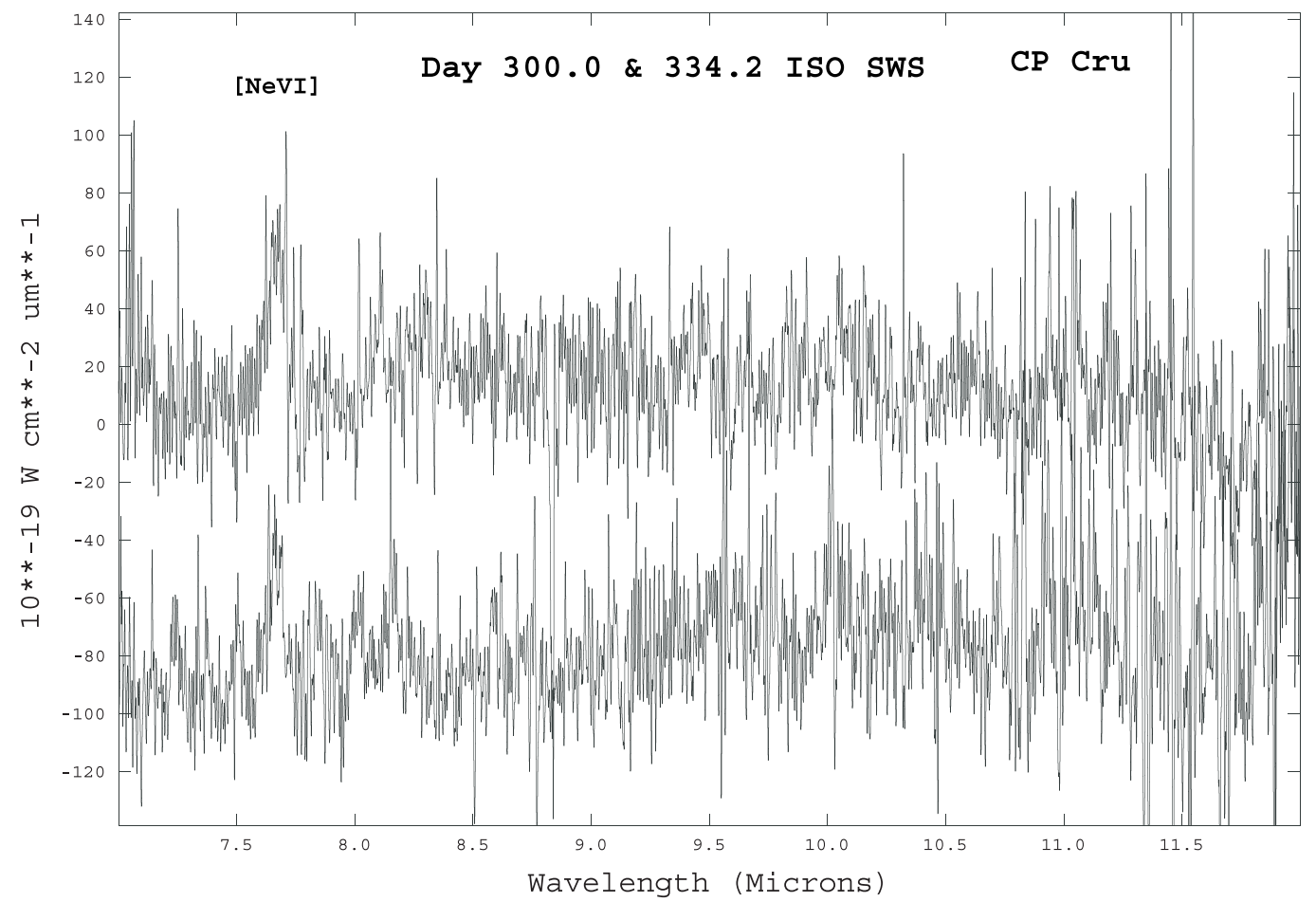

FIG. 8. - ISO SWS days 300 (top spectrum) and 334 (bottom spectrum) sample spectra showing [Ne vI] emission at $7.65 \mu \mathrm{m}$

electron temperature, $T_{e}$, and electron density, $n_{e}$ of the ejecta can be calculated. We used the [O III] $\lambda \lambda 4959+5007 /$ $\lambda 4363$ flux ratio of 20.9 , together with the [N II] $\lambda \lambda 6548+$ $6584 / \lambda 5755$ flux ratio of 0.68 and the $[\mathrm{Ne} \mathrm{v}] \lambda 3426 / 14.3 \mu \mathrm{m}$ flux ratio of 24.6 to create a plot in $n_{e}$ versus $T_{e}$ phase space (Fig. 10). The [N II] and [Ne v] diagnostic curves cross at
$T_{e}=14,000 \pm 2000 \quad \mathrm{~K}$ and $\log n_{e}=6.45 \pm 0.5 \mathrm{~cm}^{-3}$. Neither of these two curves intersects with the [O III] curve, which indicates a lower density and/or temperature for the [O III] emitting region. It should be noted that the intersection between the [N $\mathrm{NI}]$ and [Ne v] diagnostic curves in Figure 10 is rather shallow, implying significant uncertainties

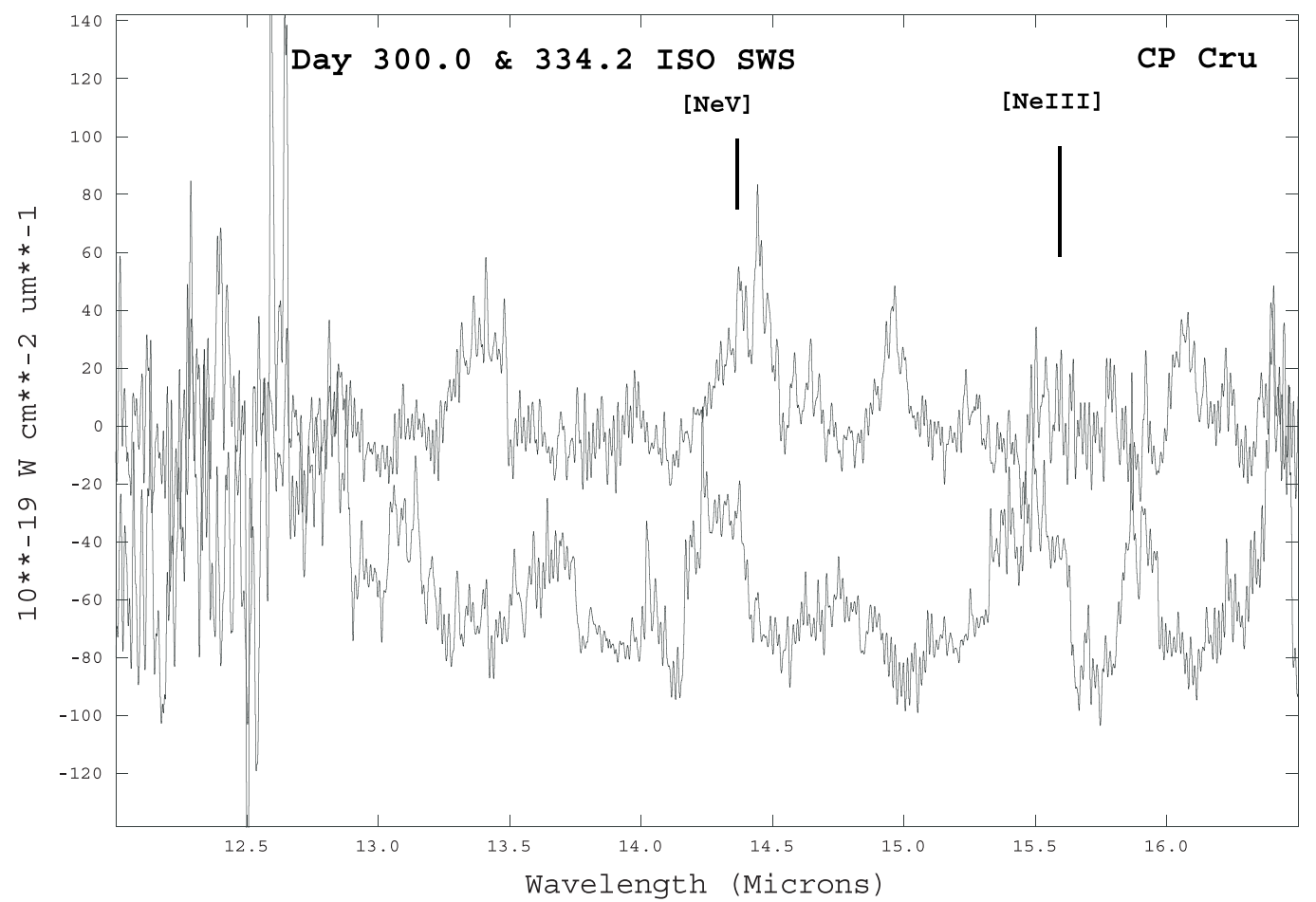

FIG. 9.- ISO SWS days 300 (top spectrum) and 334 (bottom spectrum) sample spectra showing $13-16 \mu \mathrm{m}$. Features such as those at $13.4,15.0$, and $16.0 \mu \mathrm{m}$ on day 300.0 did not appear in both scan directions. 


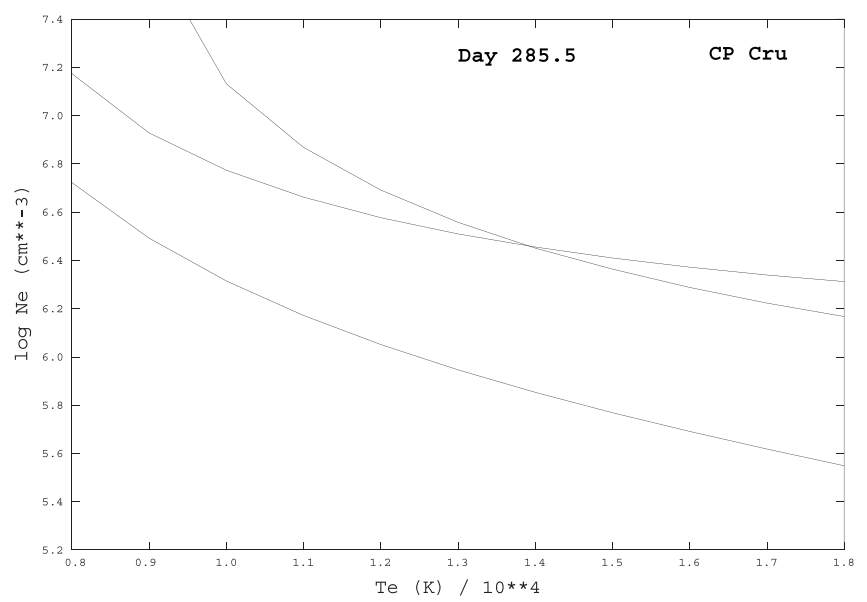

FIG. 10.-Electron temperature/density phase space. Shown are the $[\mathrm{Ne} \mathrm{v}]$ curve (solid line), the $[\mathrm{N}$ II] curve (dashed line), and the [O III] curve (dash-dotted line). The $[\mathrm{Ne} \mathrm{v}]$ and $[\mathrm{N}$ II $]$ curves cross at $T_{e}=14,000 \pm 2000$ $\mathrm{K}$ and $\log n_{e}=6.45 \pm 0.5 \mathrm{~cm}^{-3}$.

in the derived values of $T_{e}$ and $n_{e}$ if errors, such as those due to emission arising from different regions in the ejecta, are present.

\section{MODELING ANALYSIS}

Elemental abundances and other nova properties were determined for CP Cru on day 207 using the photoionization code NEBU (see Petitjean, Boisson, \& Péquignot 1990; Morisset \& Péquignot 1996; Péquignot et al. 2001) following our techniques developed in our analysis of V1425 Aql (Paper I). The number of emission lines available for modeling is somewhat smaller in CP Cru than in V1425 Aql, but requirements of smooth evolution and consistency with other dates of observation (models not shown here) introduce new constraints, leading to significant results (Péquignot et al. 2003). These constraints require a threecomponent model topologically equivalent to optically thick gas clumps, surrounded by envelopes, and embedded in a diffuse gas of somewhat lower density.

The basic properties of our best-fit model are provided in Table 5 (central source), Table 6 (shell), and Table 7 (elemental abundances).

\subsection{CP Crucis Remnant on Day 207}

The central source spectrum is described as a blackbody (temperature $T_{\mathrm{BB}}$ and luminosity $L_{\mathrm{BB}}$ ), with discontinuities

TABLE 5

Model Star Properties on Day 207

\begin{tabular}{|c|c|}
\hline Property & Quantity \\
\hline Distance $(\mathrm{pc}) \ldots \ldots \ldots$ & 2600 \\
\hline$T_{\mathrm{BB}}(\mathrm{K}) \ldots \ldots \ldots \ldots \ldots \ldots \ldots \ldots$ & $7.0 \times 10^{5}$ \\
\hline$L_{\mathrm{BB}}\left(\operatorname{ergs~s}^{-1}\right) \ldots .$. & $6.0 \times 10^{37}$ \\
\hline$f_{4 \mathrm{ryd}} \ldots \ldots \ldots \ldots \ldots \ldots$ & 0.50 \\
\hline 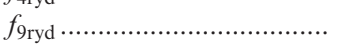 & 0.045 \\
\hline$T_{\text {eff }}(\mathrm{K})$ & $4.3 \times 10^{5}$ \\
\hline$L_{\mathrm{eff}}\left(\mathrm{ergs} \mathrm{s}^{-1}\right) \ldots \ldots \ldots \ldots \ldots \ldots \ldots$ & $8.8 \times 10^{36}$ \\
\hline$R *(\mathrm{~cm})$ & $5.9 \times 10^{8}$ \\
\hline $\log (g) \ldots \ldots$ & $\sim 8.6$ \\
\hline
\end{tabular}

TABLE 6

Model Shell Properties on Day 207

\begin{tabular}{|c|c|c|c|}
\hline Quantity & $\begin{array}{l}\text { Diffuse } \\
\text { Medium }\end{array}$ & $\begin{array}{l}\text { Clump } \\
\text { Envelopes }\end{array}$ & Clumps \\
\hline 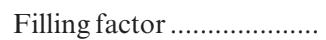 & 1.0 & 1.0 & 1.0 \\
\hline Covering factor ${ }^{\mathrm{a}} \ldots \ldots \ldots \ldots \ldots$ & 0.73 & 0.13 & 0.048 \\
\hline $\operatorname{Radius}^{\mathrm{b}}(\mathrm{cm}) \ldots \ldots$ & $4.2 \times 10^{15}$ & $4.2 \times 10^{15}$ & $4.2 \times 10^{15}$ \\
\hline Thickness $(\mathrm{cm})$ & $5.0 \times 10^{13}$ & $6.7 \times 10^{13}$ & $7.3 \times 10^{13}$ \\
\hline$\left\langle n_{\mathrm{H}}\right\rangle\left(\mathrm{cm}^{-3}\right)$ & $1.7 \times 10^{6}$ & $2.8 \times 10^{6}$ & $3.4 \times 10^{6}$ \\
\hline$\langle T\rangle(\mathrm{K}) \ldots \ldots \ldots$ & 13600 & 10200 & 9700 \\
\hline $\operatorname{Mass}\left(M_{\odot}\right) \ldots \ldots \ldots \ldots \ldots \ldots \ldots$ & $2.5 \times 10^{-5}$ & $9.7 \times 10^{-6}$ & $4.7 \times 10^{-6}$ \\
\hline Fraction $I_{\mathrm{H} \beta}$ (percent) ...... & 45 & 37 & 18 \\
\hline 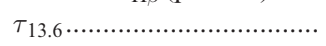 & 0.63 & 2.8 & 120. \\
\hline 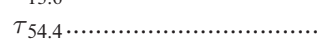 & 4.2 & 34. & 60. \\
\hline
\end{tabular}

Note.-All quantities are integrated along radii from the star.

a Distance $2.6 \mathrm{kpc}$ assumed.

${ }^{\mathrm{b}}$ Expansion velocity $2350 \mathrm{~km} \mathrm{~s}^{-1}$ assumed, see $\S 4.2$.

at the $\mathrm{He}^{+}$and $\mathrm{Ne}^{4+}$ ionization limits $(4$ and 9.3 ryd, respectively). The blackbody flux is multiplied by constant arbitrary factors $f_{4 \text { ryd }}$ between 4 and 9.3 ryd and $f_{9 \text { ryd }}$ above 9.3 ryd. Since $f_{9 \mathrm{ryd}}$ is much less than unity and $T_{\mathrm{BB}}$ is very large, the luminosity $L_{\text {eff }}$ and effective temperature $T_{\text {eff }}$ are less than the blackbody values (Table 5). The value of $L_{\text {eff }}$ is a few percent of the Eddington limit, confirming that the turnoff occurred well before day 207 and that cooling to the WD stage was very fast compared with the WD turnoff of $400 \pm 100$ days for V1425 Aql (Paper I). Note that, for both days 207 and 285 the model star radius is $6000 \mathrm{~km}$, close to the radius expected for a remnant WD.

\subsection{CP Crucis Shell on Day 207}

As for V1425 Aql, the model shell of CP Cru comprises a relatively transparent low-density medium of large covering factor in which higher density clumps, with smaller overall covering factor, are embedded and, therefore, partly shielded from the primary radiation. In an effort to obtain an optimal fit to the observed spectrum and ensure that this model is fully consistent with models determined at other dates, three different radial directions from the star are explicitly considered (Table 6): (1) radii crossing only the diffuse medium, (2) radii crossing the diffuse medium and

TABLE 7

Model Abundances on Day 207

\begin{tabular}{|c|c|c|c|}
\hline $\begin{array}{l}\text { Element } \\
\text { (1) }\end{array}$ & $\begin{array}{l}\text { By Number } \\
\text { (2) }\end{array}$ & $\begin{array}{l}\text { By Mass } \\
\text { (3) }\end{array}$ & $\begin{array}{c}\text { By Mass vs. Solar }{ }^{\mathrm{a}} \\
\text { (4) }\end{array}$ \\
\hline ....... & 1.000 & 0.474 & 0.67 \\
\hline $\mathrm{He}$ & 0.155 & 0.294 & 0.94 \\
\hline $\mathrm{C}$ & $0.7 \times 10^{-3}$ : & 0.004: & 1.3: \\
\hline $\mathrm{N}$ & $12.5 \times 10^{-3}$ & 0.083 & 75. \\
\hline $\mathrm{O}$ & $12.5 \times 10^{-3}$ & 0.095 & $10 .^{\mathrm{b}}$ \\
\hline $\mathrm{Ne}$ & $5.0 \times 10^{-3}$ & 0.047 & 27. \\
\hline ................ & 10. $\times 10^{-5}$ : & 0.0011: & 1.8: \\
\hline $\mathrm{Si}$ & $4.5 \times 10^{-5}$ & 0.0006 & 0.9 \\
\hline $\mathrm{S}$ & $3.2 \times 10^{-5}$ & 0.0005 & 1.3 \\
\hline $\operatorname{Ar}$ & $0.35 \times 10^{-5}$ & 0.00007 & 0.6 \\
\hline $\mathrm{Fe}$ & $1.2 \times 10^{-5}$ & 0.0003 & 0.3 \\
\hline
\end{tabular}

${ }^{a}$ Solar abundances from Cox 2000. 2001. 
"average" clump envelopes, and (3) radii crossing the diffuse medium, the clump envelopes, and the clump cores. This schematic model is intended to represent an "average" material. Considering material at different distances from the source is not justified, given the number of independent observables available and the good match to observations obtained with this model. On day 207, the clumps, together with their envelopes, emit about half the total $\mathrm{H} \beta$ flux. The total mass of the model shell is $3.9 \times 10^{-5} M_{\odot}$, similar to that of V1425 Aql, even though the physical conditions (and the stellar remnant properties) are quite different.

In this model, the gas density spans a maximum factor of 4 throughout the clumps and the diffuse medium. Moreover, extrapolating the day 207 and 285 model parameters back to day 24 , we find that the model correctly accounts for the intensity of [Ne II] $12.8 \mu \mathrm{m}$ observed by ISO SWS, despite the fact that the density in the ionized gas, $\left\langle n_{\mathrm{H}}\right\rangle \sim 6 \times 10^{8}$ $\mathrm{cm}^{-3}$, is more than 3 orders of magnitude larger than the critical electron density for [Ne II]. This result suggests that, contrary to a common belief, large density fluctuations are not required to explain the emission spectra of novae: (1) the coexistence of low- and high-ionization lines on the same date reflects the ionization stratification in the clumps, as depicted by photoionization models, and (2) the [Ne II] emission on day 24 reflects the large abundance of $\mathrm{Ne}^{+}$. In addition, the presence of $[\mathrm{Ne} \mathrm{VI}],[\mathrm{Si} \mathrm{VII}]$, and perhaps [Mg VIII] fine-structure lines in the spectra are correctly accounted for by the present photoionization model, in which the maximum electron temperature is $1.6 \times 10^{4} \mathrm{~K}$. Thus, no gas phase at temperature $10^{5} \mathrm{~K}$ or more is required to explain these lines. Similar conclusions are drawn from photoionization model studies of Nova Musca 1983 (Morisset \& Péquignot 1996) and V1425 Aql (Paper I).

The overall covering factor of the present model shell of CP Cru is 0.9 (Table 6). Since this factor cannot exceed unity, the model implies that the adopted distance of $2.6 \mathrm{kpc}$ is near the maximum acceptable value and is probably a rather realistic estimate of the distance, inasmuch as material was probably ejected in all directions and the shell is still relatively young and dense.

Our best-fit model also yields a shell expansion velocity of $v_{\text {exp }} \sim 2350 \mathrm{~km} \mathrm{~s}^{-1}$, larger than the value of $1650 \mathrm{~km} \mathrm{~s}^{-1}$ corresponding to the half-width at half-maximum of emission lines. We note that, although the line profiles are nearly rectangular ( $\S 3.1$ ), the line wings allow radial velocities larger than $1650 \mathrm{~km} \mathrm{~s}^{-1}$ and the line profiles tend to be double peaked (Fig. 2), suggesting that the emitting material was ejected preferentially along two opposite directions. Assuming that this preferential direction is at an angle $\geq \pi / 4$ with the line of sight, the bulk of the emitting material may be receding from the source at $2350 \mathrm{~km} \mathrm{~s}^{-1}$.

\subsection{CP Crucis Abundances}

Owing to the large temperature gradient across the shell, photoionization models are essential to derive acceptable abundances. In column (2) of Table 7, abundances derived from our photoionization models are given by number relative to $\mathrm{H}$. However, the abundances by mass (col. [3]) are more significant for this $\mathrm{H}$-deficient material. In column (4), these abundances by mass are compared with those of the solar system. With few lines detected by ISO, the model solution is more flexible than a model with more constraints. Given that, and keeping in mind the observational and theoretical uncertainties, the derived abundances may be in error by factors of $1.2(\mathrm{He}), 1.5(\mathrm{~N}, \mathrm{O}, \mathrm{Ne}$, and $\mathrm{Ar})$, and 2 ( $\mathrm{Si}, \mathrm{S}$, and $\mathrm{Fe})$, although some abundance ratios, such as $\mathrm{Ne} / \mathrm{O}$, are much more accurately determined. Abundances for $\mathrm{C}$ and $\mathrm{Mg}$ are very poorly determined. The carbon abundance is loosely constrained by radiative equilibrium. The magnesium abundance is based on [Mg VIII] $3.03 \mu \mathrm{m}$, possibly detected on day 179 . The only relatively definite conclusion concerning $\mathrm{C}$ and $\mathrm{Mg}$ is that they are not much more abundant than in the Sun. If $\mathrm{Mg}$ was as overabundant as $\mathrm{Ne}$, fine-structure lines of $\mathrm{Mg}$ would be detected easily.

Argon and iron have abundances close to solar, as expected, since these elements are not synthesized in either the parent star or the nova outburst. Considering the relatively low abundance found for iron (compare, e.g., with V1425 Aql: Paper I) and the uncertainties attached to these determinations, the most consistent overall abundance for $\mathrm{Ar}$ and $\mathrm{Fe}$ is half-solar, suggesting an old Population I origin for the binary. This conclusion also agrees with the $\mathrm{He} / \mathrm{N}$ disk nova classification from early spectra. The slight overabundance of sulfur in Table 7 is not significant.

Neon, well diagnosed by several lines from several ions, is found from models to be overabundant by a factor of 27 . The ratio $\mathrm{Ne} / \mathrm{O}$ in CP Cru is enhanced by a factor of 1.5 relative to the local ISM (and the Sun, since the solar and interstellar oxygen abundances are now identical; Allende Prieto et al. 2001). Although CP Cru should therefore be classified as a "neon nova" (often termed an "ONeMg nova" from a more theoretical standpoint), it is not clear whether existing models of standard $\mathrm{ONeMg}$ nova outbursts correctly predict the present set of abundances (e.g., Starrfield et al. 1998). First, $\mathrm{Ne} / \mathrm{O}$ is only $\sim 0.5$ by mass, whereas $\mathrm{Ne} / \mathrm{O} \geq 3$ seems more appropriate for ONeMg novae. Second, there is no obvious indication from fine-structure lines that magnesium (or silicon) may be strongly overabundant, whereas enrichments versus solar of factors of 10-100 would be expected, and sulfur is probably not enhanced either. Finally, N/O is rather large and may be quite reminiscent of $\mathrm{CO}$ novae, suggesting that the preoutburst carbon fraction was substantial in the prenova (although little carbon is left in the shell). Thus, CP Cru may be, at last, an example of the "missing link" between CO novae and ONeMg novae. Clearly, specific nova outburst models should be constructed in order to decide if the present set of abundances can be reproduced.

\subsection{Empirical Nebular Analysis}

As a consistency check with our complex NEBU photoionization model, we estimate the ionic abundances of the various elements observed in the optical and IR spectra with the assumption of uniform ejecta. We can then sum over different ionic states to estimate elemental abundances.

Using the volume emissivity ratios for hydrogen of Hummer \& Storey (1987), we derived dereddened $\mathrm{H} \beta$ fluxes for all epochs during which large-aperture hydrogen line fluxes were measured. We plot these equivalent $\mathrm{H} \beta$ fluxes as a function of time in Figure 11. The adopted intrinsic hydrogen line ratios depend only weakly upon $T_{e}$ and $n_{e}$. We adopted $T_{e}=10^{4} \mathrm{~K}$, while the $n_{e}$ values adopted for the conversion of hydrogen line fluxes to equivalent $\mathrm{H} \beta$ fluxes were obtained by extrapolating the derived day 285.5 density of $10^{6.45} \mathrm{~cm}^{-3}$ back in time assuming a $t^{-3}$ density dependence, which corresponds to a freely expanding shell 


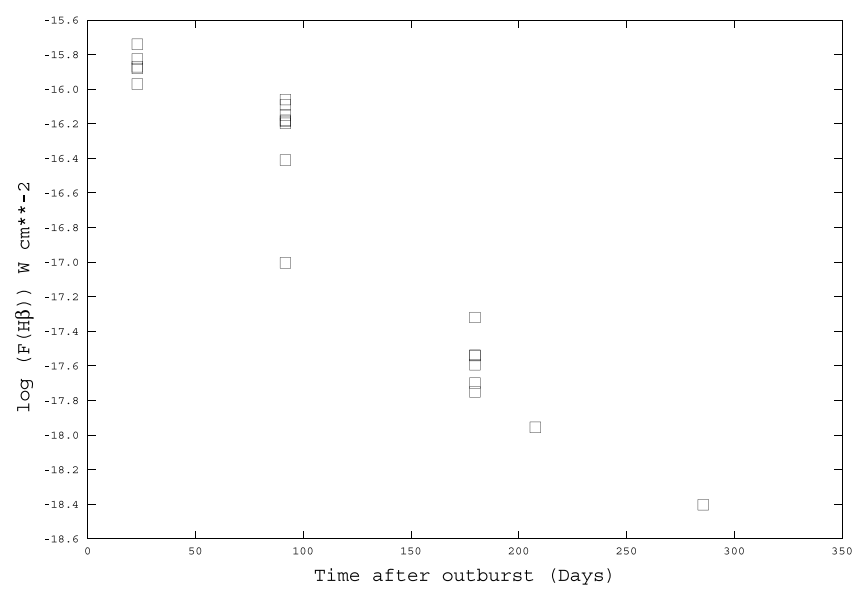

FIG. 11.-Theoretical $\mathrm{H} \beta$ temporal development. The $\mathrm{H} \beta$ fluxes are determined from other observed hydrogen lines.

in which both the shell thickness and the shell radius expand at a constant velocity. If, on the other hand, the shell thickness were to remain constant while the shell radius expanded at a constant velocity, then the mean density would fall as $t^{-2}$. For the simple case of a uniform-density shell having a constant ionized hydrogen mass [i.e., $n_{\mathrm{H}^{+}} d V=$ constant, where $V$ is volume], the flux in an optically thin hydrogen recombination line $\left(\propto n_{e} n_{\mathrm{H}^{+}} d V\right)$ should fall as $n_{e}$. From Figure 11, the observed decline of the equivalent $\mathrm{H} \beta$ flux between day 24.7 and day 285.5 can be fitted by a $t^{-2.5}$ power law, intermediate between the constant shell thickness and uniformly expanding shell thickness cases discussed above. We note from Figure 11 that different hydrogen lines measured on the same date can yield different equivalent $\mathrm{H} \beta$ fluxes, which we attribute to uncertainties in both the flux measurements and reddening corrections, as well as optical depth effects.

We determined the helium abundance using emission measures, $q_{n^{\prime}-n}$ for $\mathrm{H} \beta$ and $\mathrm{He}$ lines as given by Osterbrock (1989), Hummer \& Storey (1987), and P. J. Storey (2002, private communication) using the relation

$$
\frac{n\left(\mathrm{He}^{+p}\right)}{n\left(\mathrm{H}^{+}\right)}=\frac{I(\lambda)}{I(\mathrm{H} \beta)} \frac{q(\mathrm{H} \beta)}{q(\lambda)},
$$

where $I(\lambda) / I(\mathrm{H} \beta)$ is the observed line ratio and $q$ are
TABLE 8

Empirical He Abundances by Number

\begin{tabular}{cccc}
\hline \hline Day & $n\left(\mathrm{He}^{+}\right) / n\left(\mathrm{H}^{+}\right)$ & $n\left(\mathrm{He}^{2+}\right) / n\left(\mathrm{H}^{+}\right)$ & $n(\mathrm{He}) / n\left(\mathrm{H}^{+}\right)$ \\
\hline $91.7 \ldots \ldots \ldots \ldots \ldots$. & 0.18 & 0.04 & 0.21 \\
$179.6 \ldots \ldots \ldots \ldots \ldots$. & $0.70^{\mathrm{a}}$ & 0.03 & 0.73 \\
$207.6 \ldots \ldots \ldots \ldots$. & 0.10 & 0.07 & 0.17 \\
$266.4 \ldots \ldots \ldots \ldots \ldots$. & $\ldots$. & 0.06 & $\ldots$ \\
$285.5 \ldots \ldots \ldots \ldots$. & 0.08 & 0.06 & 0.14 \\
Average $^{\mathrm{c}} \ldots \ldots$. & $\ldots$ & $\ldots$ & $0.17 \pm 0.05$ \\
\hline
\end{tabular}

a Possible contamination with [A1 Ix] $2.04 \mu \mathrm{m}$.

${ }^{\mathrm{b}} \mathrm{No} \mathrm{He}$ I lines were strong enough to give sensible results.

c Ignoring day 179.6

emission measures, defined as $\left[4 \pi j\left(n^{\prime}-n\right)\right] /\left[n\left(\mathrm{H}^{+}\right) n_{e}\right]$ for hydrogen and with $n\left(\mathrm{H}^{+}\right)$replaced by $n\left(\mathrm{He}^{+p}\right)$ for helium. Collision effects for $\mathrm{He}$ I were accounted for using the method of Kingdon \& Ferland (1995). We report He abundances in Table 8. Using our measured and calculated $\mathrm{H} \beta$ fluxes, we determined ionic abundances with respect to $\mathrm{H}^{+}$ on days 207.6 and 285.5 using volume emissivities calculated by the IRAF STSDAS.NEBULAR.IONIC task (Shaw \& Dufour 1995) and by the multilevel statistical equilibrium code EQUIB (written by I. D. Howarth and S. Adams, unpublished):

$$
\frac{n\left(\mathrm{X}^{+p}\right)}{n\left(\mathrm{H}^{+}\right)}=\frac{I(\lambda)}{I(\mathrm{H} \beta)} \frac{j(\mathrm{H} \beta)}{j(\lambda)},
$$

where $I(\lambda) / I(\mathrm{H} \beta)$ is the observed line ratio and the $j$ 's are the calculated volume emissivities. Our estimated elemental abundances are given in Table 9. For day 285.5, we use $T_{e}=$ $14000 \pm 2000 \mathrm{~K}$ and $\log n_{e}=6.45 \pm 0.5 \mathrm{~cm}^{-3}$ as determined in $\S$ 3.3. For day 207.6, we use the same $T_{e}$, but find by extrapolation $\log n_{e}=6.86 \pm 0.5 \mathrm{~cm}^{-3}$ assuming $t^{-3}$ density dependence. We note that within the error, $n_{e}$ for both days overlap. These methods assume that the two emission lines arise in regions with similar $T_{e}$ and $n_{e}$, which may not be realistic given the clumpiness of nova ejecta (Krautter et al. 2002). However, this technique provides a first-order check of abundances determined by the photoionization model of the ejecta. Because not all ionization states were observed for all species, summing the abundances of different ions gives only a lower limit to the elemental abundances.

TABLE 9

\begin{tabular}{|c|c|c|c|c|c|c|}
\hline \multirow[b]{2}{*}{ ELEMENT } & \multicolumn{3}{|c|}{ DAY 207.6} & \multicolumn{3}{|c|}{ DAY 285.5} \\
\hline & By Number & By Mass & By Mass vs. Solar ${ }^{\mathrm{a}}$ & By Number & By Mass & By Mass vs. Solar ${ }^{a}$ \\
\hline $\mathrm{H}$ & 1.00 & 0.57 & 0.80 & 1.00 & 0.590 & 0.84 \\
\hline $\mathrm{He} . . . \ldots \ldots \ldots \ldots$ & 0.170 & 0.38 & 1.40 & 0.140 & 0.330 & 1.20 \\
\hline $\mathrm{N} \ldots \ldots \ldots \ldots \ldots$ & $1.50 \times 10^{-4}$ & $1.18 \times 10^{-3}$ & 1.1 & $1.18 \times 10^{-4}$ & $9.72 \times 10^{-4}$ & 0.9 \\
\hline $\mathrm{O} \ldots \ldots \ldots \ldots \ldots$ & $2.99 \times 10^{-3}$ & $2.70 \times 10^{-2}$ & 2.8 & $4.90 \times 10^{-3}$ & $4.62 \times 10^{-2}$ & 4.8 \\
\hline $\mathrm{Ne}^{\mathrm{b}} \ldots \ldots \ldots \ldots \ldots$ & $2.70 \times 10^{-3}$ & $3.03 \times 10^{-2}$ & $17.4^{\mathrm{c}}$ & $2.79 \times 10^{-3}$ & $3.29 \times 10^{-2}$ & $18.9^{\mathrm{c}}$ \\
\hline $\mathrm{S} \ldots \ldots \ldots \ldots \ldots$ & $3.60 \times 10^{-5}$ & $6.52 \times 10^{-4}$ & 1.8 & $1.22 \times 10^{-6}$ & $2.30 \times 10^{-5}$ & 0.06 \\
\hline $\operatorname{Ar} \ldots \ldots \ldots \ldots \ldots$ & $1.36 \times 10^{-6}$ & $3.08 \times 10^{-5}$ & $0.3^{\mathrm{d}}$ & $1.44 \times 10^{-6}$ & $3.40 \times 10^{-5}$ & $0.3^{\mathrm{d}}$ \\
\hline
\end{tabular}

EMPIRICAL AbUndances

a Solar abundances from Cox 2000. Flux-weighted average abundances taken for ions with multiple lines.

b Ne v 14.32 and Ne vi $7.65 \mu \mathrm{m}$ from ISO SWS day 300, used only for day 285.5.

${ }^{\mathrm{c}}$ Includes interpolated value for $\mathrm{Ne}^{3+}$ since the line was a possible blend with [Ar IV].

d Includes interpolated value for $\mathrm{Ar}^{3+}$. 
By mass, Ar is roughly half of the solar value, $\mathrm{S}$ is enhanced with respect to solar by about a factor of $1.5, \mathrm{~N}$ is roughly solar, $\mathrm{O}$ is enhanced by about a factor of 4 , and $\mathrm{Ne}$ is enhanced by a factor of about 18. Due to uncertainty in $T_{e}$ and $n_{e}$ and uncertainty in the line fluxes, abundance estimates are within an order of magnitude. The Ne enhancement matches well that determined by the NEBU code. However, the $\mathrm{N}$ and $\mathrm{O}$ are underestimated by large factors exposing the limitations of assuming uniform ejecta.

\section{CONCLUSIONS}

We have presented IR and optical spectroscopic observations of Nova CP Crux 1996 that cover days 24-334 post outburst. Using the expansion parallax, we determine a distance to CP Cru of $2.6 \pm 0.5 \mathrm{kpc}$. This distance implies that it reached a maximum $M_{V}=-8.7 \pm 0.5$. We have found strong [Ne II] $12.81 \mu \mathrm{m}$ emission 24 days after outburst, the earliest onset of this line in a nova. We have tracked the development of the spectrum from one of mostly $\mathrm{H}$ and $\mathrm{He}$ emission to that of a typical nebula with [O III] and Ne lines, plus "coronal" emission from $\mathrm{Ca}$ and $\mathrm{Si}$. The expansion velocity of the nova ejecta was consistent with a constant velocity over 300 days of observations. We have used the emission lines to constrain photoionization models using the NEBU code to determine abundances in the ejecta of the novae. We find $\mathrm{N}, \mathrm{O}$, and $\mathrm{Ne}$ all to be strongly enhanced by mass versus solar by factors of 75,17 , and 27 , respectively. We also find that $\mathrm{Mg}$ is loosely constrained to about solar abundance. On the basis of abundance analysis, we propose that CP Cru is an old Population I binary system. Additionally, our $\mathrm{Ne} / \mathrm{O}$ ratio of 0.5 , less than the typical 3 for an $\mathrm{ONeMg}$ nova, along with our abundances, leads us to conclude that CP Cru is an example of the intermediate population of classical novae between the canonical $\mathrm{CO}$ and $\mathrm{ONeMg}$ novae types. The properties of $\mathrm{CP}$ Cru are summarized in Table 10.
TABLE 10

Properties of CP CRUCIS

\begin{tabular}{|c|c|}
\hline Property & Value \\
\hline Discovery date .................. & 1996 August $26.04 \mathrm{UT}=\mathrm{JD} 2,450,321.54$ \\
\hline Assumed $t_{0} \ldots \ldots \ldots \ldots \ldots \ldots \ldots$ & JD 2,450,321.54 \\
\hline$m_{V_{\max }} \ldots \ldots \ldots \ldots \ldots \ldots \ldots \ldots \ldots \ldots \ldots \ldots \ldots \ldots \ldots$ & 9.25 \\
\hline 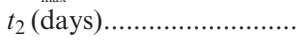 & $4.3 \pm 0.7$ \\
\hline$t_{3}$ (days) $\ldots \ldots \ldots \ldots \ldots \ldots \ldots$ & $10 \pm 2$ \\
\hline$E(B-V) \ldots \ldots \ldots \ldots \ldots \ldots \ldots \ldots \ldots \ldots$ & $1.9 \pm 0.1$ \\
\hline 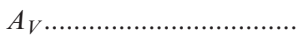 & $5.9 \pm 0.3$ \\
\hline Distance $(\mathrm{kpc}) \ldots \ldots \ldots \ldots \ldots . . . . . .$. & $2.6 \pm 0.5$ \\
\hline Ejected mass $\left(M_{\odot}\right) \ldots \ldots \ldots$ & $3.9 \times 10^{-5}$ \\
\hline Orbital period $(\mathrm{hr}) \ldots \ldots \ldots . .$. & $22.7^{\mathrm{a}}$ \\
\hline
\end{tabular}

a Woudt \& Warner 2003.

J. E. L., R. D. G., and C. E. W. were supported by an ISO block grant and the NSF. J. E. L. also acknowledges support from the University of Minnesota Graduate School. S. S. would like to acknowledge partial support to Arizona State University from NASA and NSF grants. We also thank an anonymous referee, whose comments improved our manuscript. The Infrared Space Observatory $(I S O)$ is an ESA project with instruments funded by ESA Member States (especially the PI countries: France, Germany, the Netherlands, and the United Kingdom) and with the participation of Institute of Space and Aeronautical Science and NASA. The ISO Spectral Analysis Package (ISAP) is a joint development of the LWS and SWS Instrument Teams and Data Centers. Contributing institutes are Centre d'Etude Spatiale Rayonnements, Institut d'Astrophysique Spatiale, Infrared Processing and Analysis Center, Max Planck Institute, Rutherford Appleton Laboratory, and Space Research Organization of the Netherlands.

\section{REFERENCES}

Allende Prieto, C., Lambert, D. L., \& Asplund, M. 2001, ApJ, 556, L63

Cox, A. N. 2000, Allen's Astrophysical Quantities (4th ed.; New York: Springer)

de Graauw, Th., et al. 1996, A\&A, 315, L49

Della Valle, M., \& Benetti, S. 1997, IAU Circ. 6532

Della Valle, M., \& Livio, M. 1998, ApJ, 506, 818

Downes, R. A., \& Duerbeck, H. W. 2000, AJ, 120, 2007

Evans, A., et al. 2003, AJ, submitted

Ford, H. C., \& Ciardullo, R. 1988, in ASP Conf. Ser. 4, The Extragalactic

Distance Scale, ed. S. van den Burgh \& C. J. Prichet (San Francisco: ASP), 128

Garradd, G. J. 1996, IAU Circ. 6468

Gehrz, R. D., Truran, J. W., Williams, R. E., \& Starrfield, S. 1998, PASP, 110,3

Harris, M. J., Naya, J. E., Teegarden, B. J., Cline, T. L., Gehrels, N.,

Palmer, D. M., Ramaty, R., \& Seifert, H. 1999, ApJ, 522, 424

Hayward, T. L., et al. 1996, ApJ, 469, 854

Howarth, I. D. 1983, MNRAS, 203, 301

Hummer, D. G., \& Storey, P. J. 1987, MNRAS, 224, 801

Kingdon, J., \& Ferland, G. J. 1995, ApJ, 442, 714

Krautter, J., et al. 2002, AJ, 124, 2888

Liller, W., Overbeek, D., \& Mattei, J. 1996, IAU Circ. 6463

Lyke, J. E., et al. 2001, AJ, 122, 3305

Monard, B., Nelson, P., \& Farrell, F. 1996, IAU Circ. 6466
Morisset, C., \& Péquignot, D. 1996, A\&A, 312, 135

Osterbrock, D. E. 1989, Astrophysics of Gaseous Nebulae and Active Galactic Nuclei (Sausalito: Univ. Science Books)

Payne-Gaposchkin, C. 1964, The Galactic Novae (New York: Dover)

Péquignot, D., et al. 2001, in ASP Conf. Ser. 247, Spectroscopic Challenges of Photoionized Plasmas, ed. G. Ferland \& D. W. Savin (San Francisco: ASP), 533

2003, in preparation

Petitjean, P., Boisson, C., \& Péquignot, D. 1990, A\&A, 240, 433

Rieke, G. H., \& Lebofsky, M. J. 1985, ApJ, 288, 618

Salama, A., Evans, A., Eyres, S. P. S., Leech, K., Barr, P., \& Kessler, M. F. 1996, A\&A, 315, L209

Salama, A., Eyres, S. P. S., Evans, A., Geballe, T. R., \& Rawlings, J. M. C. 1999, MNRAS, 304, L20

Shaw, R. A., \& Dufour, R. J. 1995, PASP, 107, 896

Shortridge, K. 1993, in ASP Conf. Ser. 52, Astronomy Data Analysis Software and Systems II, ed. R. J. Hanish, R. J. V. Brissenden, \& J. Barnes (San Francisco: ASP), 219

Starrfield, S., Truran, J. W., Wiescher, M. C., \& Sparks, W. M. 1998, MNRAS, 296, 502

Sturm, E. 1997, Ap\&SS, 258, 285

Valentijn, E. A., et al. 1996, A\&A, 315, L60

Williams, R. E. 1992, AJ, 104, 725

Woudt, P. A., \& Warner, B. 2003, MNRAS, 340, 1011 